\title{
Sensitivity and specificity considerations for fMRI encoding, decoding, and mapping of auditory cortex at ultra-high field
}

Citation for published version (APA):

Moerel, M., De Martino, F., Kemper, V. G., Schmitter, S., Vu, A. T., Uğurbil, K., Formisano, E., \& Yacoub, E. (2018). Sensitivity and specificity considerations for fMRI encoding, decoding, and mapping of auditory cortex at ultra-high field. Neuroimage, 164, 18-31. https://doi.org/10.1016/j.neuroimage.2017.03.063

Document status and date:

Published: 01/01/2018

DOI:

10.1016/j.neuroimage.2017.03.063

Document Version:

Publisher's PDF, also known as Version of record

\section{Document license:}

Taverne

Please check the document version of this publication:

- A submitted manuscript is the version of the article upon submission and before peer-review. There can be important differences between the submitted version and the official published version of record.

People interested in the research are advised to contact the author for the final version of the publication, or visit the DOI to the publisher's website.

- The final author version and the galley proof are versions of the publication after peer review.

- The final published version features the final layout of the paper including the volume, issue and page numbers.

Link to publication

\footnotetext{
General rights rights.

- You may freely distribute the URL identifying the publication in the public portal. please follow below link for the End User Agreement:

www.umlib.nl/taverne-license

Take down policy

If you believe that this document breaches copyright please contact us at:

repository@maastrichtuniversity.nl

providing details and we will investigate your claim.
}

Copyright and moral rights for the publications made accessible in the public portal are retained by the authors and/or other copyright owners and it is a condition of accessing publications that users recognise and abide by the legal requirements associated with these

- Users may download and print one copy of any publication from the public portal for the purpose of private study or research.

- You may not further distribute the material or use it for any profit-making activity or commercial gain

If the publication is distributed under the terms of Article $25 \mathrm{fa}$ of the Dutch Copyright Act, indicated by the "Taverne" license above, 


\title{
Sensitivity and specificity considerations for fMRI encoding, decoding, and mapping of auditory cortex at ultra-high field
}

\author{
Michelle Moerel $^{\mathrm{a}, \mathrm{b}, \mathrm{c}, \mathrm{d}, *}$, Federico De Martino ${ }^{\mathrm{a}, \mathrm{c}, \mathrm{d}}$, Valentin G. Kemper ${ }^{\mathrm{c}, \mathrm{d}}$, Sebastian Schmitter ${ }^{\mathrm{a}, \mathrm{e}}$, \\ An T. Vu ${ }^{a, f, g}$, Kâmil Uğurbil ${ }^{\mathrm{a}}$, Elia Formisano ${ }^{\mathrm{b}, \mathrm{c}, \mathrm{d}}$, Essa Yacoub ${ }^{\mathrm{a}}$ \\ a Center for Magnetic Resonance Research, Department of Radiology, University of Minnesota, Minneapolis, USA \\ b Maastricht Centre for Systems Biology, Maastricht University, Maastricht, The Netherlands \\ ${ }^{\mathrm{c}}$ Department of Cognitive Neuroscience, Faculty of Psychology and Neuroscience, Maastricht University, Maastricht, The Netherlands \\ d Maastricht Brain Imaging Center (MBIC), Maastricht, The Netherlands \\ e Department of Biomedical Magnetic Resonance, Physikalisch-Technische Bundesanstalt, Berlin, Germany \\ ${ }^{\mathrm{f}}$ Helen Wills Neuroscience Institute, University of California, Berkeley, CA, USA \\ g Advanced MRI Technologies, Sebastopol, CA, USA
}

\section{A R T I C L E I N F O}

\section{Keywords:}

Ultra-high field fMRI

Sensitivity

Specificity

Human auditory cortex

\begin{abstract}
A B S T R A C T
Following rapid technological advances, ultra-high field functional MRI (fMRI) enables exploring correlates of neuronal population activity at an increasing spatial resolution. However, as the fMRI blood-oxygenation-leveldependent (BOLD) contrast is a vascular signal, the spatial specificity of fMRI data is ultimately determined by the characteristics of the underlying vasculature. At 7 T, fMRI measurement parameters determine the relative contribution of the macro- and microvasculature to the acquired signal. Here we investigate how these parameters affect relevant high-end fMRI analyses such as encoding, decoding, and submillimeter mapping of voxel preferences in the human auditory cortex. Specifically, we compare a $\mathrm{T}_{2}{ }^{*}$ weighted fMRI dataset, obtained with 2D gradient echo (GE) EPI, to a predominantly $\mathrm{T}_{2}$ weighted dataset obtained with 3D GRASE. We first investigated the decoding accuracy based on two encoding models that represented different hypotheses about auditory cortical processing. This encoding/decoding analysis profited from the large spatial coverage and sensitivity of the $\mathrm{T}_{2}{ }^{*}$ weighted acquisitions, as evidenced by a significantly higher prediction accuracy in the GEEPI dataset compared to the 3D GRASE dataset for both encoding models. The main disadvantage of the $\mathrm{T}_{2}{ }^{*}$ weighted GE-EPI dataset for encoding/decoding analyses was that the prediction accuracy exhibited cortical depth dependent vascular biases. However, we propose that the comparison of prediction accuracy across the different encoding models may be used as a post processing technique to salvage the spatial interpretability of the GE-EPI cortical depth-dependent prediction accuracy. Second, we explored the mapping of voxel preferences. Large-scale maps of frequency preference (i.e., tonotopy) were similar across datasets, yet the GE-EPI dataset was preferable due to its larger spatial coverage and sensitivity. However, submillimeter tonotopy maps revealed biases in assigned frequency preference and selectivity for the GE-EPI dataset, but not for the 3D GRASE dataset. Thus, a $\mathrm{T}_{2}$ weighted acquisition is recommended if high specificity in tonotopic maps is required. In conclusion, different fMRI acquisitions were better suited for different analyses. It is therefore critical that any sequence parameter optimization considers the eventual intended fMRI analyses and the nature of the neuroscience questions being asked.
\end{abstract}

\section{Introduction}

At ultra-high magnetic fields, functional MRI (fMRI) benefits from increased blood-oxygenation-level-dependent (BOLD)-based susceptibility contrast (Ogawa et al., 1992; Yacoub et al., 2001) and a higher signal-to-noise ratio (SNR; Vaughan et al., 2001), allowing the acquisition of images at an increased spatial resolution. $3 \mathrm{~T}$ fMRI experiments are typically run with $2-3 \mathrm{~mm}$ isotropic resolutions, whereas at $7 \mathrm{~T}$ millimeter to submillimeter spatial resolutions are not uncommon. This has attracted substantial interest from the neuros-

\footnotetext{
* Correspondence to: Maastricht Centre for Systems Biology (MaCSBio), Maastricht University, P.O. Box 616, 6200 MD Maastricht, The Netherlands.

E-mail addresses: michelle.moerel@maastrichtuniversity.nl (M. Moerel), f.demartino@maastrichtuniversity.nl (F. De Martino), valentin.kemper@maastrichtuniversity.nl (V.G. Kemper), sschmitt@umn.edu (S. Schmitter), anvu@berkeley.edu (A.T. Vu), kamil@cmrr.umn.edu (K. Uğurbil), e.formisano@maastrichtuniversity.nl (E. Formisano), yaco0006@umn.edu (E. Yacoub).
} 
cientific community, as the higher spatial resolution allows exploring the human brain non-invasively at an unprecedented spatial scale. Specifically, a submillimeter isotropic resolution could allow investigations of functional processing separately in deep, middle, and superficial cortical depths. As different cortical depths have characteristic patterns of anatomical connectivity and unique functional roles (Douglas and Martin, 2004; Larkum, 2013), the possibility of obtaining non-invasive laminar functional data is intriguing (Kok et al., 2016; Muckli et al., 2015; Olman et al., 2012). Moreover, the cortical depthdependent exploration of functional responses to stimulus features (e.g., visual orientation, or sound frequency) enables examining the stability of feature preference throughout cortical depth (Zimmermann et al., 2011; De Martino et al., 2015; Nasr et al., 2016). This may provide information regarding the existence and functional role of columnar feature organizations, a neuroscientific topic that has been debated and unresolved to date (Horton and Adams, 2005; Rakic, 2008).

Simply acquiring higher resolution fMRI images does not guarantee a corresponding increase in the specificity of the measured functional processes, as the BOLD signal reflects the hemodynamic response to neural activity. The spatial specificity of the BOLD signal is therefore constrained by the underlying vasculature. Importantly, fMRI data can be acquired in various ways at ultra-high fields, impacting the overall sensitivity of the signals to different components of the vasculature. The most common approach is to use gradient-echo echo-planar imaging (GE-EPI), a $\mathrm{T}_{2}{ }^{*}$ weighted sequence. The acquired GE-EPI signal contains contributions both from macro- and microvasculature (Uğurbil et al., 2003; Uludağ et al., 2009; Yacoub et al., 2005). The macrovasculature includes large diving veins penetrating the gray matter (GM) and the large pial veins situated on top of the GM (Duvernoy et al., 1981). These large draining vessels produce a strong BOLD effect (i.e., a high sensitivity), while also decreasing the spatial specificity as they generate responses away from the actual site of neuronal activity (e.g., higher signal towards the GM surface; Goense et al., 2007; Harel et al., 2006; Kok et al., 2016; Koopmans et al., 2011, 2010; Polimeni et al., 2010; Ress et al., 2007; Siero et al., 2011; Zhao et al., 2004). Several approaches have previously been used in order to minimize the contribution of large and unspecific veins to GE-EPI data. The first approach involves excluding the voxels closest to veins from the analysis. The superficial cortical layers may be excluded altogether, or venous voxels may be identified and excluded (Koopmans et al., 2010; Lu et al., 2010; Shmuel et al., 2007). As a second approach, post processing techniques have been used to suppress the BOLD signal of large veins after acquisition. For example, the complex fMRI signal (both magnitude and phase) can be used to estimate and remove the part of the signal that originated from the macrovasculature (Menon, 2002; Vu and Gallant, 2015). A third approach can be used if a priori information about the representation of the stimulus on the cortex is available. Such a priori information may be used to identify voxels with spatially non-specific responses. These voxels can then be excluded from the analysis, and the remainder of the GE-EPI dataset can be used to map high resolution organizations (Muckli et al., 2015; Yacoub et al., 2007).

Alternatively, at high fields it is also possible to collect submillimeter $\mathrm{T}_{2}$ weighted functional images (for example using a spin echo (SE) sequence). Such a $\mathrm{T}_{2}$ weighted sequence suppresses the contribution of large vessels (Yacoub et al., 2003), and thus the acquisitions have relatively more signal from smaller veins. This results in signals with high spatial specificity (De Martino et al., 2013b; Duong et al., 2003; Goense et al., 2007; Harel et al., 2006; Uludağ et al., 2009; Zhao et al., 2004), which are ideal for laminar and columnar investigations of the human cortex. Indeed, in a direct comparison, SE was shown to be more specific than GE in identifying ocular dominance columns in human primary visual cortex (V1; Yacoub et al., 2007).

While $\mathrm{T}_{2}$ weighted $\mathrm{SE}$ sequences are superior in terms of spatial specificity, they also suffer from several drawbacks. Their acquisition time is longer, measured BOLD contrast is lower (lower sensitivity), and specific absorption rate (SAR) is higher compared to $\mathrm{T}_{2}{ }^{*}$ weighted sequences. As such, to make submillimeter $\mathrm{T}_{2}$ weighted acquisitions a realistic option for high field fMRI applications, significant tradeoffs are made in the imaging field of view (FOV) and the volume coverage (i.e., data can be collected from only a small region of the brain). For 2D SEEPI this has led to acquisitions with a single thick slice and a small in plane FOV (Yacoub et al., 2008, 2007). More recently, to overcome some of the limitations of SE-EPI, such as relatively low functional contrast to noise (CNR) and the anisotropic voxel resolution, 3D inner volume gradient and spin echo (3D GRASE) was proposed as an alternative (Feinberg et al., 2008; Oshio and Feinberg, 1991). Using a train of refocusing pulses to encode a 3D slab, combined with inner volume selection, 3D GRASE provides a predominantly $\mathrm{T}_{2}$ weighted signal (Kemper et al., 2015a). However, in order to keep the acquired signal predominantly $\mathrm{T}_{2}$ weighted and reduce the spatial blurring along the phase encode directions (partition and in-plane phase encode), the field of view (FOV) is still limited (Kemper et al., 2015b). Further, while the measured BOLD CNR with 3D GRASE is higher than with a SE-EPI sequence (Kemper et al., 2015a), it is still substantially smaller than the BOLD contrast measured with GE-EPI (De Martino et al., 2013b). In spite of these constraints, the imaging advantages of the 3D GRASE approach permitted the first columnar mapping study in humans outside of V1 (Zimmermann et al., 2011), and the first human columnar mapping study outside of visual cortex (De Martino et al., 2015).

Given the advantages and disadvantages of both $\mathrm{T}_{2}{ }^{*}$ and $\mathrm{T}_{2}$ weighted acquisitions, currently there is no generic answer as to the optimal strategy for exploring laminar and columnar processing in the human cortex at high magnetic fields. Here, we directly compared a $\mathrm{T}_{2}{ }^{*}$ weighted sequence (multislice GE-EPI) to a predominantly $\mathrm{T}_{2}$ weighted sequence (3D GRASE), both acquired at $0.8 \mathrm{~mm}$ isotropic, in a study of the human auditory cortex of six volunteers at $7 \mathrm{~T}$. We conducted two investigations of cortical depthdependent functional processing. First, we compared the performance of the two datasets following a decoding analysis. fMRI decoding assesses the information present in a brain region or subset of voxels, and can thus also be used to quantify the information present as a function of cortical depth (Muckli et al., 2015). We based sound decoding on two different computational encoding models which reflect different hypotheses about auditory cortical processing (Moerel et al., 2012; Santoro et al., 2014). Second, we compared the two datasets with respect to sound feature mapping (i.e., estimation of the voxels' tonotopic frequency preference and selectivity). We interpret results in light of the voxels' proximity to large veins.

Our results suggest that different fMRI acquisitions are well suited for different analyses. Compared to the 3D GRASE dataset, the GE-EPI dataset had the advantage of higher prediction accuracy in the encoding/decoding analyses. While prediction accuracy in the GEEPI dataset increased towards the pial surface, we propose that the difference between the encoding models' performances can be used as a post processing technique that salvages the interpretability of the GEEPI cortical depth-dependent prediction accuracy. While large-scale maps of frequency preference (i.e., tonotopy) showed no observable bias, cortical depth-dependent tonotopic maps displayed a bias in the assigned best frequency preference and selectivity in the GE-EPI dataset only. Thus, the mapping of cortical feature preference organized at this fine spatial scale benefitted from the high specificity of the 3D GRASE dataset. In conclusion, at ultra-high fields, where a choice between signals with high CNR dominated by large draining veins or signals with lower CNR dominated by the microvasculature is possible, it is essential that the choice of imaging sequence considers the intended subsequent fMRI analyses.

\section{Material and methods}

\section{Ethics statement}

The experimental procedures were approved by the Institutional 
Review Board (IRB) for human subject research at the University of Minnesota. Informed consent was obtained from each participant before commencement of the measurements.

\section{Subjects}

Six healthy volunteers participated in this study (mean age [SD] $=28.5$ [7.8]; two males and four females). The subjects had no history of hearing disorder or neurological disease.

\section{Experimental design and stimuli}

The study involved three experimental sessions, in which all subjects participated. The first session served to collect high resolution anatomical data for the purpose of segmentation, cortical layer sampling (Zimmermann et al., 2011), and vessel segmentation. In the second and third session, high resolution GE-EPI and 3D GRASE data were collected, respectively, while subjects listened to natural sounds. One subject participated in a fourth session, during which a Time of Flight (TOF) dataset and susceptibility-weighted images (SWI) were acquired. The TOF and SWI images served to create an angiogram and venogram respectively, used for validating the vessel segmentation procedure.

The natural sounds included recordings of the following sound categories: speech, voices, animal cries, music, tools, and nature scenes (144 sounds in total; 24 sounds per category). Sounds were sampled at $16 \mathrm{kHz}$ and their duration was $1000 \mathrm{~ms}$. Sound onset and offset were ramped with a $10 \mathrm{~ms}$ linear slope, and their energy (RMS) levels were equalized. Sounds were presented to the subjects in the MRI scanner using the MRI-compatible S14 model earbuds of Sensimetrics Corporation (www.sens.com) with a linear frequency transfer up to $8 \mathrm{kHz}$.

The natural sounds were divided into 4 non-overlapping sets of 36 sounds each. These sets were created randomly under the constraint that all semantic categories should be equally represented in each set. Sessions 2 and 3 consisted of 12 and 16 fMRI data collection runs, respectively. We presented one stimulus set (36 distinct sounds) per run, and each set was presented three or four times across the full session (i.e., 3 or 4 runs per sound set for sessions 2 and 3, respectively). Stimuli were presented at a jittered inter-stimulus interval of 2, 3, or 4 TRs. Sounds were played, with additional random jitter, in silent gaps between functional volume acquisitions. Trials where no sound was presented were added ( $8 \%$ of the trials). Subjects were instructed to attend to the sounds and perform a one-back task. That is, they indicated with a button press if the exact same sound was played on consecutive trials (occurring on $6 \%$ of the trials; repeat trials were not considered in the training and testing of encoding models).

\section{MRI acquisition}

All measurements were performed on a $90 \mathrm{~cm}$ bore $7 \mathrm{~T}$ whole body magnet (Magnex Scientific, Abingdon, UK) driven by a Siemens console (Siemens Medical Systems, Erlangen) using a custom whole head 32 channel loop transceiver and a high performance head gradient insert. In the first session, $\mathrm{T}_{1}$, proton density [PD], and $\mathrm{T}_{2}{ }^{*}$ weighted data with full brain coverage were collected at a voxel size of $0.6 \mathrm{~mm}$ isotropic. Two $\mathrm{T}_{1}$ weighted scans were acquired using a modified magnetization-prepared rapid gradient-echo (MPRAGE) sequence. PD datasets were acquired with the same MPRAGE as the $\mathrm{T}_{1}$ weighted image but without the inversion recovery module. See Table 1 for the acquisition parameters.

In the second session, GE-EPI images were acquired (see Table 1 for the acquisition parameters). Slice placement included the bilateral auditory cortex. This session consisted of 12 runs, each $\sim 5$ min in duration. In session two and three, a $\mathrm{T}_{1}$ weighted scan was acquired for the purpose of realignment across sessions and slice placement.
The third session served to collect high-resolution 3D GRASE images (see Table 1). Due to the limited FOV of the 3D GRASE settings, slice placement included Heschl's gyrus (HG) and regions at its anterior and posterior adjacency. In $3 / 6$ subjects these regions were covered bilaterally, and in the other subjects only the left hemisphere was covered. This session consisted of 16 runs, each $\sim 4$ min in duration.

TOF and SWI data was collected from an oblique slab oriented along the lateral sulcus, which covered the auditory cortex bilaterally. Parameters of the TOF and SWI datasets are listed in Table 1.

\section{Anatomical data analysis}

PD images were used to minimize receive coil inhomogeneities in $\mathrm{T}_{1}$ weighted images (Van de Moortele et al., 2009; left column of Fig. 1). The resulting $T_{1} / P D$ datasets were further corrected for residual inhomogeneities, and down-sampled to $0.8 \mathrm{~mm}$ isotropic resolution matching the resolution of the functional data. Automatic tools of BrainVoyager QX were used to detect the white matter (WM)$\mathrm{GM}$, and GM-cerebrospinal fluid (CSF) boundaries. Both boundaries were manually edited to ensure a correct definition of the regions of interest (HG and regions at its anterior and posterior adjacency).

Next, cortical thickness was measured as implemented in BrainVoyager QX 2.8 (Brain Innovation, Maastricht, Netherlands), which uses a procedure based on the Laplace equation (De Martino et al., 2013b; Jones et al., 2000; Zimmermann et al., 2011). In brief, this procedure defines the intensity values at the inner and outer gray matter boundary (i.e., the WM-GM and GM-CSF boundary, respectively), and smooths the intensity values of voxels between these boundaries. The solution of Laplace's equation creates a smooth field that allows calculating a gradient at each voxel. Integrating gradients across voxels results in 'streamlines' that define the maximum change (i.e., the shortest path) between the WM-GM and GM-CSF boundaries. Based on this cortical thickness computation, cortical depth-dependent grids are defined. Note that the Laplace equation is only used to the compute cortical thickness. Instead, the cortical depth-dependent grids are spaced equidistantly across cortical depth, rather than as being defined by the Laplace solution. That is, the first regularly spaced grid is created orthogonal to, and halfway through, the streamlines (cortical thickness=0.5). Additional grids (closer to the WM, or to the CSF) are created by moving up or down along the streamlines to get corresponding grid points at other cortical depths (left column of Fig. 1). For each subject and hemisphere, we created a grid containing nine (ranging from 0.1 [close to WM] to 0.9 [close to CSF] cortical thickness) cortical depths. The WM-GM and GM-CSF boundaries as defined on the anatomical data are not included in these grids.

Vessels were identified by selecting the darkest voxels in the $\mathrm{T}_{2}{ }^{*}$ and SWI datasets, and the brightest voxels in the TOF dataset, followed by manual corrections (right column of Fig. 1). Separately for each dataset (i.e., separately for the $\mathrm{T}_{2}{ }^{*}$, SWI, and TOF data), the Euclidean distance between each gridpoint and all voxels identified as vessels was computed. Each gridpoint's distance to a vessel was assigned as the minimum distance (in $\mathrm{mm}$; i.e., the distance to the closest vessel). Correspondence between the $\mathrm{T}_{2}{ }^{*}$, SWI, and TOF-based vessel segmentation was assessed with a correlation analysis.

\section{Functional data analysis}

The functional data were analyzed with BrainVoyager QX and custom MATLAB (The MATHWORKS Inc., Natick, MA, USA) code. Preprocessing consisted of slice scan-time correction (with sinc interpolation), 3-dimensional motion correction, temporal high pass filtering (removing drifts of 2 cycles and less per run), and temporal smoothing of the time series (2 data points). Functional data were co-registered to the anatomical data, the result of which was reviewed in each subject and adjusted where needed. Next, the data were 
Table 1

Acquisition parameters.

\begin{tabular}{|c|c|c|c|c|c|c|c|}
\hline & TR (ms) & TI (ms) & Gap (ms) & TE (ms) & Echo spacing (ms) & Flip angle $\left({ }^{\circ}\right)$ & GRAPPA \\
\hline T1 & 3100 & 1500 & na & 3.5 & na & 4 & 3 \\
\hline PD & 2160 & na & na & 3.5 & na & 4 & 3 \\
\hline $\mathbf{T}_{2}{ }^{*}$ & 3700 & na & na & 16 & na & 4 & 3 \\
\hline GE-EPI & 2400 & na & 1200 & 22.8 & 0.7 & 90 & 3 \\
\hline 3D GRASE & 2000 & na & 310 & 29.2 & 0.7 & $90 ; 180$ & na \\
\hline TOF & 25 & na & na & 3.3 & na & 22 & 2 \\
\hline \multirow[t]{2}{*}{ SWI } & 28 & na & na & 14.4 & na & 15 & 2 \\
\hline & MB & Matrix size & slices & Voxel resolution & averages & TA (min) & \\
\hline T1 & na & $384 \times 384$ & 256 & $0.6 \mathrm{~mm}$ & 2 & 14 & \\
\hline PD & na & $384 \times 384$ & 256 & $0.6 \mathrm{~mm}$ & 1 & 5 & \\
\hline $\mathbf{T}_{2}{ }^{*}$ & na & $384 \times 384$ & 256 & $0.6 \mathrm{~mm}$ & 1 & 8 & \\
\hline GE-EPI & 2 & $232 \times 256$ & 36 & $0.8 \mathrm{~mm}$ & na & 5 per run & \\
\hline 3D GRASE & na & $28 \times 250$ & 16 & $0.8 \mathrm{~mm}$ & na & 4 per run & \\
\hline TOF & na & $452 \times 576$ & 80 & $0.4 \mathrm{~mm}$ & 1 & 6 & \\
\hline SWI & na & $432 \times 576$ & 80 & $0.4 \mathrm{~mm}$ & 2 & 12 & \\
\hline
\end{tabular}

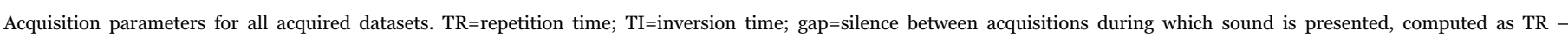

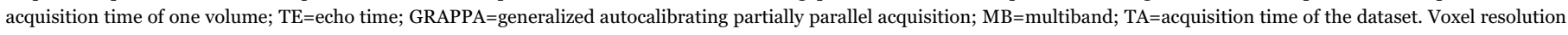
was isotropic for all datasets.

brought to ACPC space (i.e., rotation to bring the line between the anterior and posterior commissure horizontal). Overall percent signal change (PSC) maps were computed based on the responses to natural sounds and sampled at different cortical depths (using the grids described in 'Anatomical data analysis'). The relation between PSC and distance to veins was assessed by grouping gridpoints per cortical depth according to their distance to a vein (4 groups, $25 \%$ of gridpoints per group for each cortical depth), and computing PSC separately for each group. We tested for a significant correlation between PSC and distance to veins using a non-parametric analysis, implemented in order to correctly deal with the fact that 6 out of 9 data points came from the same three subjects. That is, we performed $2^{\text {NumberOfSubjects }}$ permutations of the sign of the data to generate a null distribution, where the data coming from the same subject was kept together across permutations. The p-value was assigned as the number of permutations that resulted in a correlation further away from zero than the original data divided by the total number of permutations.

\section{Comparison of computational model performance across datasets}

After exploring the cortical depth-dependent PSC and its relation to veins in the GE-EPI and 3D GRASE dataset, we next tested how these cortical depth-dependent patterns affected the performance of decoding based on two different encoding models in the two datasets. We chose to test two encoding models, as we were not only interested in overall decoding performance but also wished to explore how cortical depth-dependent variations in PSC would interact with the performance across encoding models. The following two encoding models were tested (for details, see Moerel et al., 2012; Santoro et al., 2014): a simple frequency model, and a model that defined voxel responses in terms of their frequency-specific modulation tuning (i.e., tuning to temporal modulation rates and spectral modulation scales). The representation of the sounds in the frequency model space was obtained as the output of the first (early) stage of a biologically inspired model of auditory processing (Chi et al., 2005; NSL Tools package, available at http://www.isr.umd.edu/Labs/NSL/Software.htm). This model mimics the spectral transformation of sounds passing through the cochlea to the midbrain, and includes a bank of 128 overlapping bandpass filters equally spaced along a logarithmic frequency axis (180-7040 Hz; range of 5.3 octaves). To represent sounds by the frequency model, the spectrograms resulting as the output of this model were averaged over time resulting in 128 model parameters to estimate.

For the modulation model, the output of the first stage of the NSL model was passed to a second model stage. This stage mimics cortical auditory processing by extracting the modulation content from the sounds' spectrograms using a set of modulation filters (temporal modulation frequencies of $\omega=[1,39,27] \mathrm{Hz}$; spectral modulation frequencies $\Omega$ of $=[0.5,1,2,4]$ cycles/octave). The filter output was computed at each frequency bin, and then averaged over time. To limit the number of (correlated) features, we divided the frequency axis in 8 bins with equal bandwidth in octaves, and averaged the modulation energy within each frequency bin. This resulted in 128 model parameters ( 8 frequencies $\times 4$ temporal modulation rates $\times 4$ spectral modulation scales) to estimate. In summary, each computational model represents the sounds in an $[S \times F]$ feature matrix $\mathbf{W}$, where $S$ is the number of natural sounds and $F$ is the number of features to estimate.

The model parameters were estimated in 4-fold cross-validation, where each cross-validation consisted of 108 training sounds $S_{\text {train }}$ and 36 testing sounds $S_{\text {test }}$. Per cross-validation, we computed the response to training sounds for each voxel $i, Y_{\text {train, } i}\left[S_{\text {train }} \times 1\right]$ as follows. The data were first denoised (Kay et al., 2013; http:// kendrickkay.net/GLMdenoise/) and then a voxel-wise hemodynamic response function (HRF) common to all sounds was estimated (Kay et al., 2008a). This HRF was used to compute a beta weight for each sound (see Moerel et al., 2015, 2012 for details). The response for each voxel $i$ to testing sounds, $Y_{\text {test }, i}\left[S_{\text {test }} \times 1\right]$, was computed following the same procedure but used the HRF as estimated on the training sounds.

The response to the sounds in voxel $i$ was modeled as a linear transformation of the feature matrix $\mathbf{W}$ :

$Y_{\text {train }, i}=\mathbf{W}_{\text {train }} R_{i}$

where $\mathbf{W}_{\text {train }}\left[S_{\text {train }} \times F\right]$ is the representation of the training sounds in the model space, and $R_{i}$ is an $[F \times 1]$ vector of model parameters whose elements $R_{i j}$ quantify the contribution of feature $j$ to the overall response of voxel $i$. Eq. (1) was solved using ridge regression (Hoerl and Kennard, 1970a). The regularization parameter $\lambda$ was determined independently for each voxel by automatically inspecting the stability of the ridge trace (Hoerl and Kennard, 1970b; Santoro et al., 2014). We assessed model performance as its accuracy in predicting responses to novel testing sounds ("sound identification analysis"; Kay et al., 2008b; Moerel et al., 2013; Santoro et al., 2014). Namely, we used the estimated feature preference of each voxel $i$ to predict the response $\hat{Y}_{\text {test }, i}$ as:

$\hat{Y}_{\text {test }, i}=\mathbf{W}_{\text {test }} R_{i}$

where $\mathbf{W}_{\text {test }}\left[S_{\text {test }} \times F\right]$ is the representation of the testing sounds in the model space.

We evaluated model prediction by computing a sound identification score. This score expresses to what extent a model could accurately 

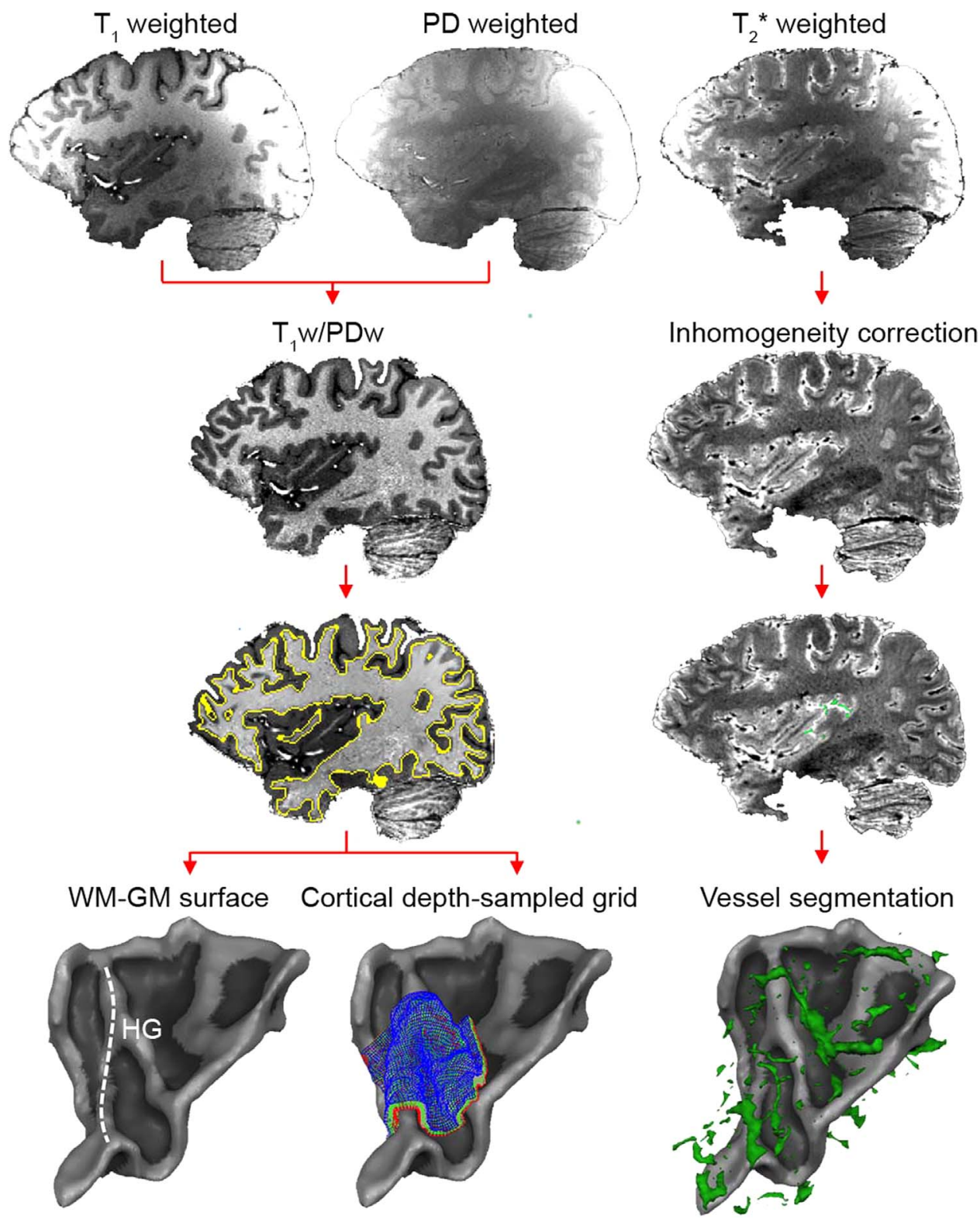

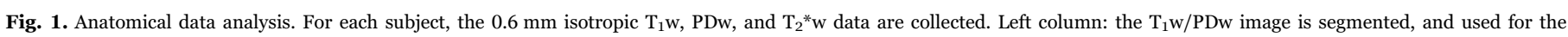
creation of WM-GM surfaces and cortical depth-dependent grids. Right column: the $\mathrm{T}_{2}{ }^{*} \mathrm{w}$ image is used to identify vessels.

predict a specific testing sound from the pool of all testing sounds. The sound identification score is computed as follows. For each sound $k$ we horizontally concatenated the predicted response $\hat{Y}_{\text {test }, k}[1 \times V]$ across cross-validations, and computed its correlation to the measured fMRI responses to all testing sounds $\mathbf{Y}_{\text {test }}\left[S_{\text {test }} \times V\right]$ horizontally concatenated across cross-validations. Rank $r_{k}$ of the correlation between predicted and observed responses to sound $k$ measures the models ability to correctly match predicted response $\hat{Y}_{\text {test }, k}[1 \times V]$ with measured response $Y_{\text {test }, k}[1 \times V]$. Thus, a rank of 1 indicates perfect prediction, while a rank of $S_{\text {test }} / 2$ represents chance, and $S_{\text {test }}$ (where the number of testing sounds=36) represents the worst outcome. The rank was normalized between 0 and 1 . Prediction accuracy $P_{k}$ of each sound was defined as 1 - the normalized rank:

$P_{k}=1-\frac{r_{k}-1}{S_{\text {test }}-1}$

Values of $P_{k}$ range between 0 and 1, with perfect prediction=1 and chance $=0.5$. The overall accuracy of the model was obtained as the mean prediction accuracy across all testing sounds. Models and datasets were statistically compared by performing a two-way repeated measures ANOVA after Fisher transformation of the prediction accuracy values.

Cortical depth-dependent model performance was assessed by sampling matrices $\mathbf{Y}$ and $\mathbf{R}$ at 9 cortical depths (using the grids described in 'Anatomical data analysis'), and repeating the analysis procedure per cortical depth. Statistical significance was assessed for deep, middle, and superficial cortical depths (0.2, 0.5, and 0.8 cortical depth, respectively). Separately for GE EPI and 3D GRASE, a two-way repeated measures Analysis of Variance (ANOVA) with factors 'Computational Model' and 'Cortical Depth' after Fisher transformation of the prediction accuracy values was performed.

The relationship between computational model performance and distance to veins was assessed by grouping gridpoints per cortical depth according to their distance to a vein (4 groups, $25 \%$ of gridpoints per group for each cortical depth), and computing the models' prediction accuracy separately for each group. 


\section{Comparison of estimated voxel preferences}

Next, we evaluated the mapping of the voxels' frequency preference (i.e., tonotopy) in detail. Large-scale tonotopy maps were created by color-coding each voxel according to the frequency with the highest weight in the trained modulation model (the voxel's best frequency [BF]). A red-yellow-green-blue color scale was used, where preference for low and high frequencies was assigned to red and blue colors, respectively. GE-EPI and 3D GRASE tonotopy maps were smoothed with a Gaussian kernel (FWHM=4 voxels; map smoothing applied on this volume-based analysis only; all cortical depth dependent analyses were performed on unsmoothed maps), and correlated to each other. Significance of observed correlations between the tonotopic maps as defined by the two datasets was assigned based on permutation testing ( $N=200$; null-distribution consists of the correlation between maps after imposing the same map smoothness on randomized 3D GRASE maps).

For the left hemisphere only, we computed group tonotopy maps by bringing the anatomical and functional data to Talairach space and performing a cortex-based alignment (CBA; Goebel et al., 2006) on the anatomical WM-GM surfaces. Functional maps were sampled on these CBA surfaces. Group maps display the mean preferred frequency for those vertices that were included in the map of at least 3 subjects.

We compared the specificity of cortical depth dependent tonotopic maps as computed on the GE-EPI and 3D GRASE datasets. To this end, the weights of the trained modulation model were sampled at different cortical depths. As for the large-scale tonotopy maps, each gridpoint's $\mathrm{BF}$ was assigned as the frequency with the highest weight in the trained modulation model. The relationship between BF and cortical depth was assessed by plotting the BF histogram separately for each cortical depth. Following the computation of tuning width in animal electrophysiology (Q-value; Cheung et al., 2001; Imaizumi et al., 2004) and previous fMRI studies (De Martino et al., 2013a; Moerel et al., 2012), the tuning width (TW; i.e., the selectivity of the gridpoint's frequency preference) of each gridpoint $g$ was computed by fitting a Gaussian curve (with its mean constrained to the gridpoint's BF) to the voxel's frequency weights, computing $\mathrm{TW}_{g}=\mathrm{BF}_{g} / \mathrm{FWHM}_{g}$ (where $\mathrm{FWHM}_{g}=$ full width at half maximum of the best fitting Gaussian curve). Note that high and low values of TW indicate narrow and broad frequency tuning, respectively. In deep, middle and superficial cortical depths, the difference in TW across datasets was tested for significance with a non-parametric analysis. Specifically, $2^{\text {NumberOfSubjects }}$ permutations of the data labels ('GE EPI' or '3D GRASE') were performed to generate a null distribution. In these permutations, the data coming from the same subject was kept together. The p-value was assigned as the number of permutations that resulted in a larger difference in TW than the original data, divided by the total number of permutations.

Finally, we assessed the relationship between the correspondence in tonotopy maps across datasets and distance to veins. For each gridpoint, we computed the difference in BF (in octaves) as assigned by the GE-EPI and 3D GRASE dataset. Per cortical depth, the gridpoints were grouped according to their distance to a vein (10 groups, $10 \%$ of gridpoints per group for each cortical depth). In deep, middle and superficial cortical depths, the average difference in BF across datasets was compared between the group closest and furthest from veins with a non-parametric analysis. Specifically, $2^{\text {NumberOfSubjects }}$ permutations of the data labels ('close to veins' or 'far from veins') were performed to generate a null distribution. In these permutations, the data coming from the same subject was kept together. The p-value was assigned as the number of permutations that resulted in a larger difference between locations close to and far from veins than the original data, divided by the total number of permutations.

\section{Results}

Cortical depth-dependent responses to the natural sounds

We observed significant responses to the sounds on HG, Heschl's sulcus, and parts of planum polare (PP), planum temporale (PT) and the superior temporal gyrus (STG) in both the GE-EPI and 3D GRASE dataset ( $q$ [FDR] $<0.05$; see Supplementary Fig. 1). Due to limitations in the 3D GRASE coverage, bilateral auditory cortex was only covered in $3 / 6$ subjects. In the remaining subjects, only the left auditory cortex was included. For both datasets, results are reported for 9 hemispheres (6 left hemispheres and 3 right hemispheres).

We examined the percent signal change (PSC) across cortical depths by sampling the data on the cortical depth-dependent grids. Note that due to the grid sampling, the same subset of voxels was analyzed across datasets. Overall, PSC was lower for 3D GRASE than for GE-EPI. This magnitude was to be expected as the BOLD signal in auditory cortex is much lower than in visual cortex, and the 3D GRASE percent signal change can be expected to be less than $50 \%$ of that of the GE-EPI (De Martino et al., 2013b). In spite of the low PSC, the observation of significant responses to the sounds (see Supplementary Fig. 1) indicates that the lower responses in GRASE are accompanied by a relatively low variability. While PSC was constant across cortical depths for the 3D GRASE dataset, PSC increased towards the surface for the GE-EPI dataset. This pattern was present at the level of individual subjects (Fig. 2a), and was preserved when averaging across 9 hemispheres (Fig. 2b).

Next, we analyzed the relationship between a gridpoint's PSC and its distance to a vein. As a first step, we validated our $\mathrm{T}_{2}{ }^{*}$-based vessel segmentation procedure against standard methods of angiogram or venogram creation (i.e., vessel segmentation on a SWI and TOF dataset, respectively). Fig. 3a shows matched slices from the TOF, SWI, and $\mathrm{T}_{2}{ }^{*}$ datasets. As expected, low intensity voxels in the SWI dataset (reflecting veins) corresponded to low intensity voxels in the $\mathrm{T}_{2}{ }^{*}$ dataset. Both surface veins located on top of the GM and diving veins could be observed (Fig. 3a, red circles and arrows, respectively). This correspondence between the datasets was confirmed quantitatively. Gridpoint distances to a vessel (in mm; Fig. 3b) were negatively correlated when defined on the SWI and TOF datasets (average Fisher's transformed correlation across cortical depth [SE] $r=-0.26$ [0.016]), confirming that these datasets identify different vessels (veins and arteries, respectively; Fig. 3c). Distances to vessels defined by the SWI and $\mathrm{T}_{2}{ }^{*}$ datasets were positively correlated (average Fisher's transformed correlation across cortical depth [SE] $r=0.44$ [0.037]), confirming that they both identified veins (Fig. 3c).

When overlaying the $\mathrm{T}_{2}{ }^{*}$-based venogram with the maps of PSC, a visual correspondence between vein locations and highest PSC was evident (Fig. 4). This correspondence was most noticeable in the GEEPI dataset. Quantification of the correspondence between distance to a vein (in $\mathrm{mm}$ ) and PSC showed a significant negative correlation for GE-EPI (average Fisher's transformed correlation [SE] $r=-0.34$ [0.030]; non-parametric permutation analysis; $p=0.016$ ), confirming that locations with a lower distance (i.e., closer) to veins had a higher PSC. No significant correlation was present in the 3D GRASE dataset (average Fisher's transformed correlation [SE] $r=-0.01$ [0.052]). We further explored the cortical depth dependency of PSC by dividing the gridpoints in four groups according to their distance to veins (see Supplementary Fig. 2). For GE-EPI, the cortical depth dependency of PSC was stronger for gridpoints closest to veins (red line in Fig. 2c; increase from 0.83 PSC close to the WM to 1.87 PSC close to the CSF) than for gridpoints furthest from veins (blue line in Fig. 2c; increase from 0.49 PSC close to the WM to 0.88 PSC close to the CSF). However, even for gridpoints furthest from veins (blue line in Fig. 2c), an increase of PSC towards the GM-CSF surface could still be observed. Moreover, the proximity to veins changed PSC also in deep GM (0.10.3 cortical depth).

\section{Encoding and decoding performance}

As a next step, we analyzed the GE-EPI and 3D GRASE datasets using on a model-based decoding analysis. For both the tonotopy and 

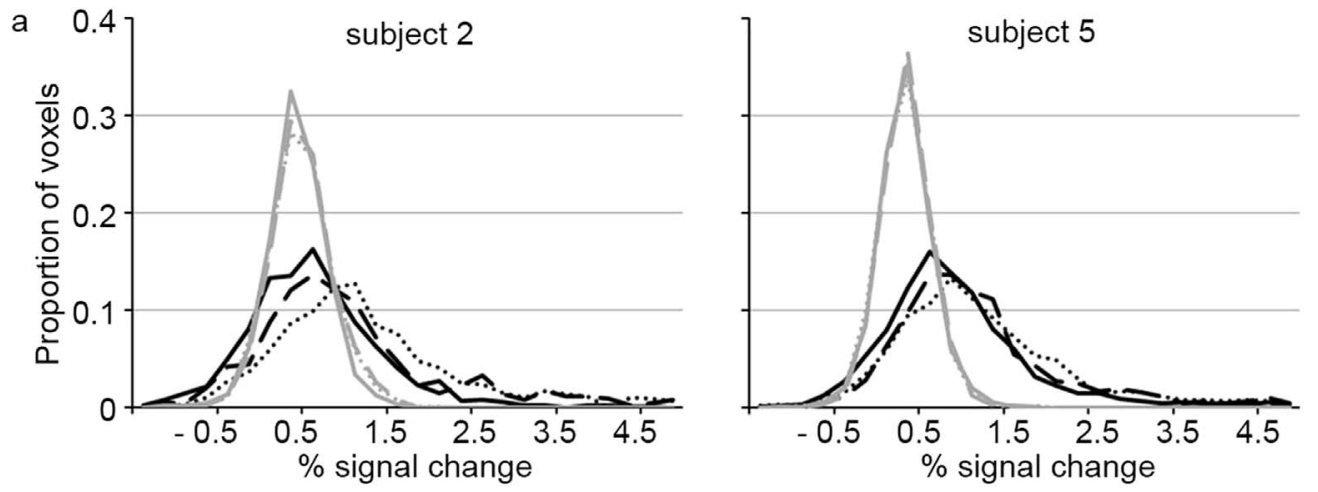

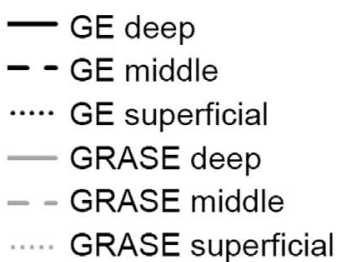

Distance to vein

$0.85-1.56 \mathrm{~mm}$

$1.02-2.20 \mathrm{~mm}$

$1.32-2.67 \mathrm{~mm}$

$1.84-3.34 \mathrm{~mm}$
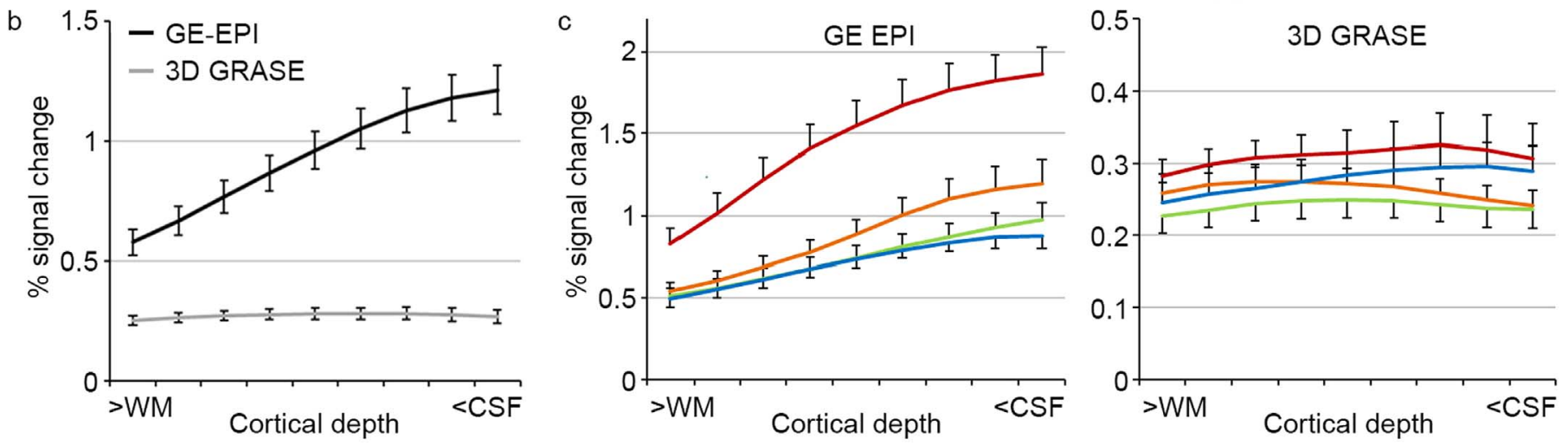

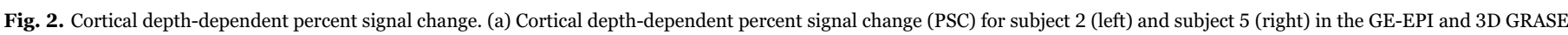

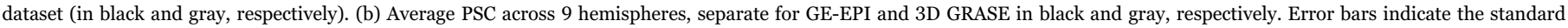

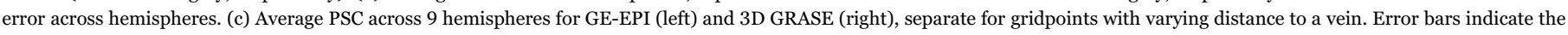

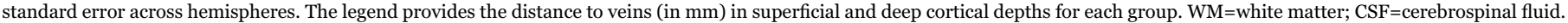

the modulation model, we examined prediction accuracy throughout the superior temporal plane, irrespective of cortical depth (including all voxels that responded significantly to sounds $[p<0.05$ uncorrected]; voxels selected per dataset). The modulation model significantly outperformed the simpler tonotopy model across datasets (two-way repeated measures ANOVA on Fisher transformed values, on 'Model' [tonotopy, modulation] and 'Method' [GE-EPI (all), 3D GRASE (all)]; no significant interaction; main effect of 'Model'; $F[1,5]=52.43$; $p=0.0008$ ). Overall prediction accuracy was significantly higher in the GE-EPI than 3D GRASE dataset (Fig. 5a: compare 'GE-EPI (all)' to '3D GRASE (all)'; main effect of 'Method'; F[1,5]=22.54; $p=0.0051$ ). To further explore the origin of the prediction accuracy difference between the GE-EPI and 3D GRASE dataset, we repeated the analysis of the GEEPI dataset while limiting the number of voxels in two ways. First, when selecting only those voxels within the FOV of the 3D GRASE dataset, overall prediction accuracy for GE-EPI decreased (Fig. 5a: compare 'GE-EPI (all)' to 'GE-EPI (FOV GRASE)'), but was significantly higher than that of the 3D GRASE dataset (two-way repeated measures ANOVA on Fisher transformed values; no significant interaction; main effect of 'Model'; F[1,5]=17.07; $p=0.0091$; main effect of 'Method'; $\mathrm{F}[1,5]=8.74 ; p=0.032)$.Second, we computed the prediction accuracy on the GE-EPI dataset using the same number of voxels as selected in the 3D GRASE dataset (within the FOV of the 3D GRASE dataset; Fig. 5a: 'GE-EPI (nvox GRASE)'). Overall prediction accuracy for GE-EPI was still significantly higher than that of the 3D GRASE dataset (two-way repeated measures ANOVA on Fisher transformed values; no significant interaction; main effect of 'Model'; $F[1,5]=19.50$; $p=0.0069$; main effect of 'Method'; $\mathrm{F}[1,5]=12.58 ; p=0.016$ ).

We next analyzed prediction accuracy per cortical depth (Fig. 5b and c). For the GE-EPI dataset (Fig. 5b), prediction accuracy increased towards the CSF for both models (two-way repeated measures ANOVA on Fisher transformed values, on 'Model' [tonotopy, modulation] and
'Cortical depth' [deep, middle, superficial]; no significant interaction; significant main effect of 'Model'; $\mathrm{F}[1,5]=12.55 ; p=0.017$, and main effect of 'Cortical depth'; $\mathrm{F}[2,10]=10.63 ; p=0.0033$ ). While the modulation model outperformed the tonotopy model across cortical depths in the 3D GRASE data, the prediction accuracy was instead stable across cortical depths, irrespective of the model (Fig. 5c; two-way repeated measures ANOVA on Fisher transformed values; no significant interaction; no significant main effect of 'Cortical depth'; significant main effect of 'Model'; $\mathrm{F}[1,5]=8.02 ; p=0.037$ ). Thus, while only the GE-EPI dataset showed a significant increase in prediction accuracy with cortical depth, the difference in the model performance was the same across datasets (Fig. 5d). In the GE-EPI dataset only, the voxels closest to veins (with the highest PSC) had the highest prediction accuracy (Fig. 5e). The experimental effect (i.e., the difference in computational model performance), however, was not related to vein distance (Fig. 5f).

\section{Specificity of cortical depth-dependent tonotopic maps}

We compared the mapping of frequency preference (i.e., tonotopy) based on the trained modulation model across datasets. While small differences between tonotopic maps across datasets were observed, on a large spatial scale these maps were significantly similar across datasets in 5 out of 6 subjects (average correlation across subjects [Fisher's transformation] $r=0.35 ; p<0.005$ in 5 subjects based on permutation testing). Their large-scale pattern was in accordance with previously reported tonotopy maps. That is, maps contained a low frequency cluster on $\mathrm{HG}$, bordered postero-medially and anterolaterally by preference for high frequencies (first column of Fig. 6 shows tonotopic maps in an individual, and Supplementary Fig. 3 shows tonotopy group maps; Da Costa et al., 2011; Formisano et al., 2003; Moerel et al., 2012; Saenz and Langers, 2014). 

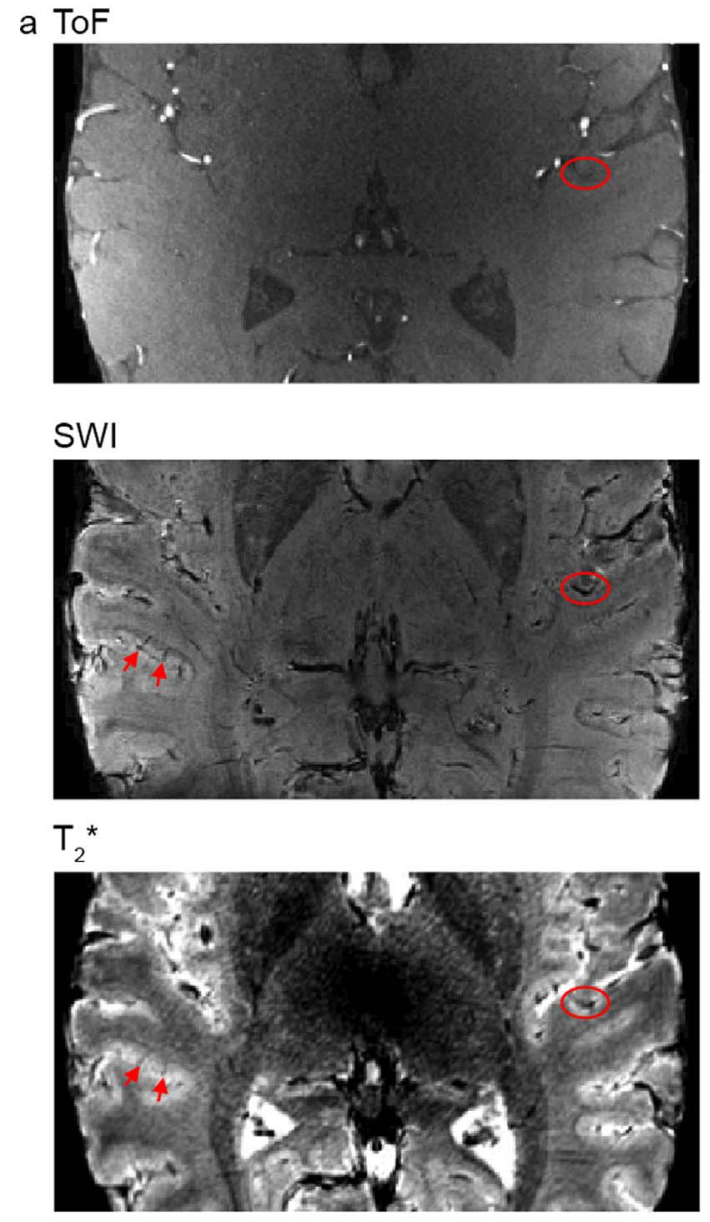

b Close to WM
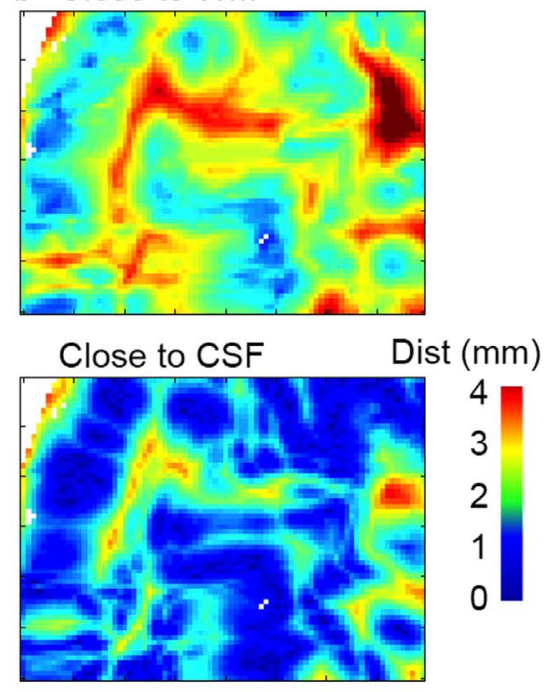

c Correlation across datasets

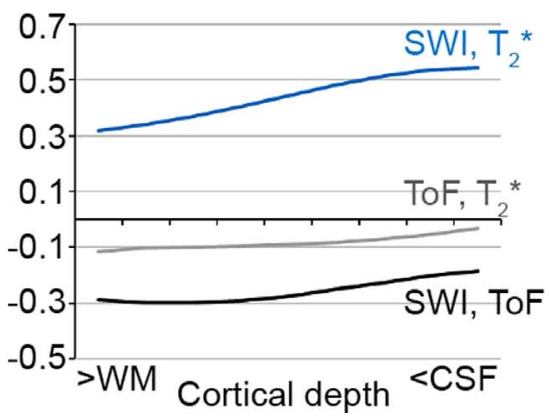

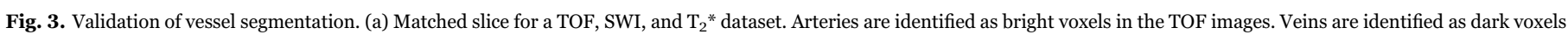

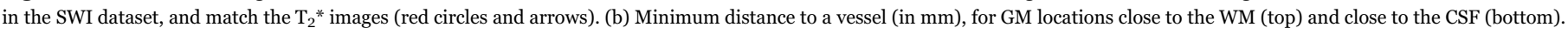

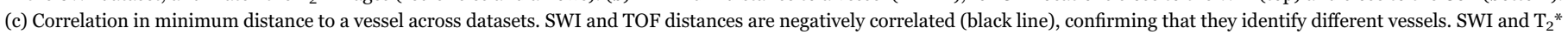
are positively correlated (blue line), confirming that they both identify veins. GM=gray matter; WM=white matter; CSF=cerebrospinal fluid.

Next, we explored tonotopy maps separately for deep, middle, and superficial cortical depths. Fig. 6 shows cortical depth-dependent tonotopy maps for a representative subject. While the BF across the cortex was stable throughout deep, middle, and superficial cortical depths in the 3D GRASE dataset, the overall BF in this particular subject decreased towards superficial cortical depths (i.e., maps became more red) in the GE-EPI dataset. For other subjects/hemispheres, both increases and decreases in overall BF were observed. This suggests the presence of a bias in BF in the GE-EPI dataset.

Three analyses support the presence of a bias in the estimated frequency tuning in the GE data. First, across hemispheres, the histogram of the gridpoints' BF broadens toward the CSF in GE-EPI, indicating a shift in frequency preference (Fig. $7 \mathrm{a}$ and b). Second, to test if biases in frequency preference were related to a gridpoint's distance to large veins, the difference between the GE-EPI and 3D GRASE tonotopy maps (in octaves) was computed separately for voxels close to and far from veins. The difference between the GE-EPI and 3D GRASE tonotopy maps (in octaves) was significantly higher in gridpoints close to veins than far from veins (Fig. $7 \mathrm{c}$ ). This effect was marginally significant in middle cortical depths (non-parametric permutation analysis, $p=0.078$ ), and significant in superficial cortical depths (non-parametric permutation analysis, $p=0.016$ ). Finally, we tested if frequency selectivity (i.e., gridpoint's tuning width) was biased in the GE-EPI and 3D GRASE dataset. The tuning width (TW) of voxels increased toward the cortical surface in the GE-EPI dataset only (Fig. 7d). TW was significantly higher in GE-EPI than 3D GRASE for both middle and superficial cortical depths (non-parametric permuta- tion analysis, $p=0.047$ and $p=0.031$ for middle and superficial cortical depths, respectively).

\section{Discussion}

At ultra-high fields, because of the high functional CNR, there are several options as to how fMRI data can be acquired. Based on the data and analyses presented here, we argue that the optimal choice in sequence and acquisition parameters depends on the nature of the neuroscience question being asked and on the subsequent planned analyses.

Cortical depth-dependent sound responses and their spatial relation to large vessels

We explored the difference in cortical depth-dependent responses across the GE-EPI and 3D GRASE dataset by investigating their dependence on proximity to veins. Given the spatial resolution of the anatomical data, resulting venograms were likely sensitive to large pial veins (typically 1-4 mm in diameter; Duvernoy et al., 1981) and surely missed a large number of smaller (e.g., diving) veins. In the GE-EPI dataset, locations closer to large veins identified by the venograms displayed higher BOLD responses. This relationship between vein distance and GE-EPI PSC was strongest at the GM surface, where we observed more than a twofold increase in response strength when comparing locations close to and far from veins. The correspondence between vein distance and response strength diminished towards the 

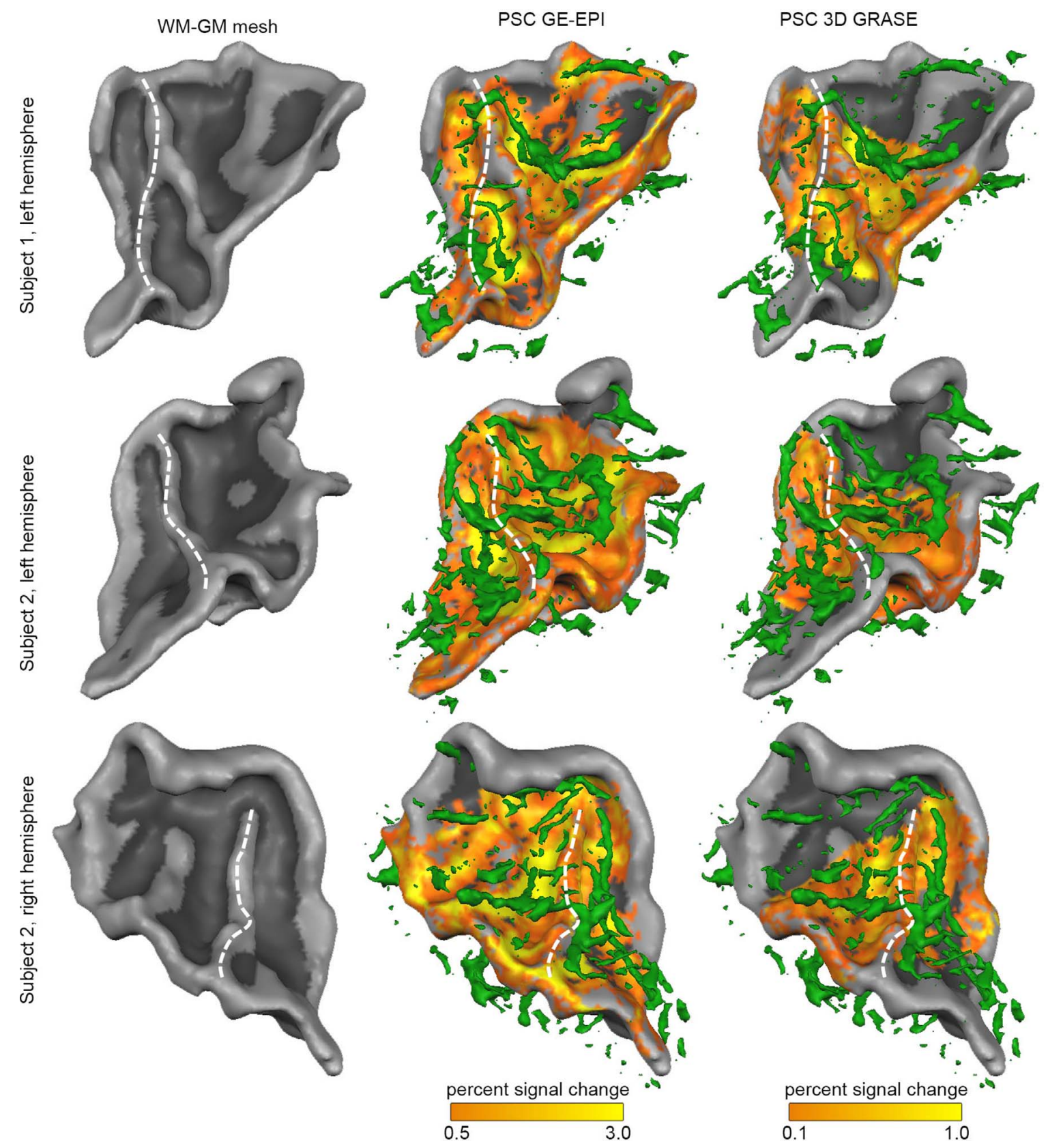

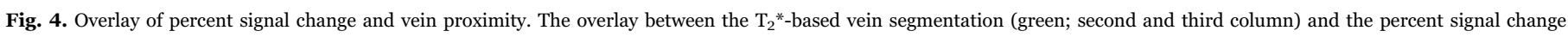

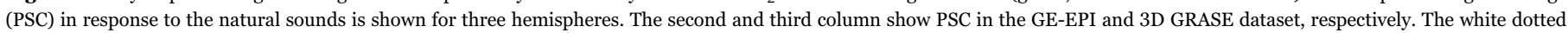
line indicated the location of $\mathrm{HG}$.

WM. Interestingly, though, while the dependence between PSC and distance to veins decreased it did not disappear in deep GM. That is, even in deep GM (i.e., close to WM), functional responses were substantially larger in locations close to veins than in locations further from veins (1.7 fold increase). Even in the $25 \%$ of gridpoints furthest from veins, response strength increased towards the GM surface (1.8 fold increase).

As a large number of veins could not be identified with the spatial resolution of our measurements, our results likely underestimated the effect of veins on PSC, prediction accuracy, and map specificity. It would be of interest to extend this work at higher spatial resolution (possibly in animal models; Yu et al., 2016) to more completely estimate the effect of veins. Importantly, even while underestimating the effect of veins, our results show that while the dependence between PSC and distance to superficial veins decreased it did not disappear in deep GM. Thus, while "large vein" effects can be reduced using post- processing techniques that remove voxels in close proximity to vessels (Ahveninen et al., 2011; Menon, 2002; Polimeni et al., 2010; Yacoub et al., 2001), our results demonstrate that large vein contaminations affect every cortical depth in a GE-EPI dataset.

\section{Coverage and PSC beneficial for encoding and decoding analyses}

How does the large vein contribution to the GE-EPI dataset affect the results of the fMRI analysis? Across datasets, the modulation model significantly outperformed the simpler tonotopy model. This is in accordance with our previous work (Santoro et al., 2014), and confirms that auditory cortical processing can be well described by the frequency-specific tuning of auditory cortical neuronal populations to joint temporal and spectral modulations. While the datasets give the same 'neuroscientific' answer, we furthermore observed that prediction accuracy was higher for GE-EPI than for 3D GRASE. Part of this 

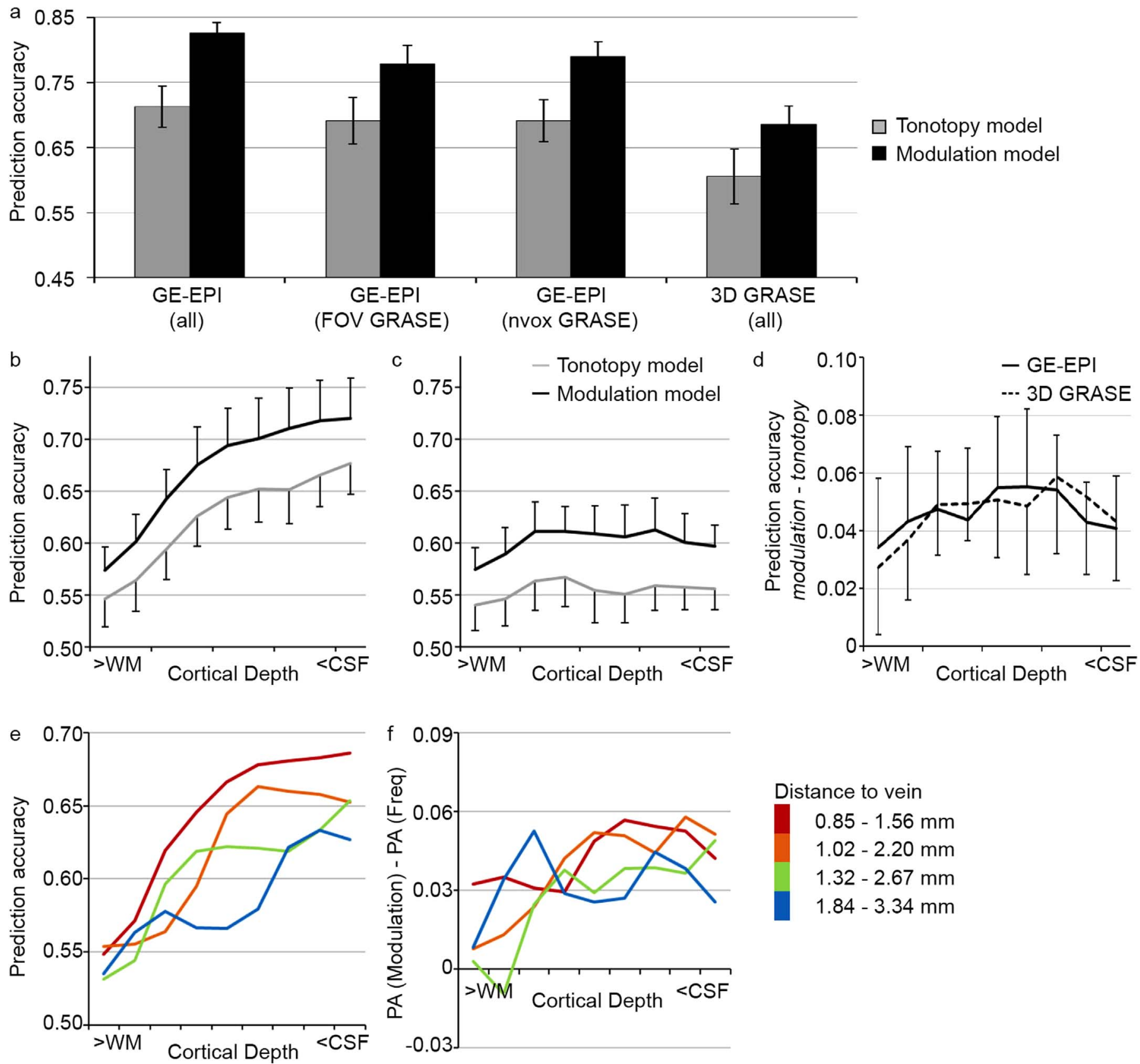

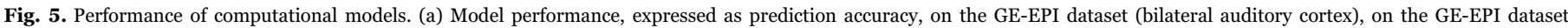

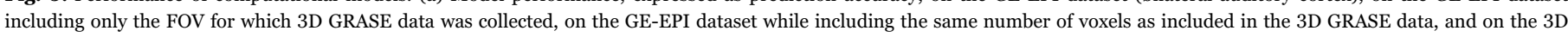

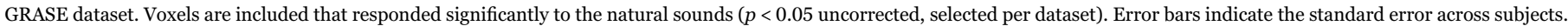

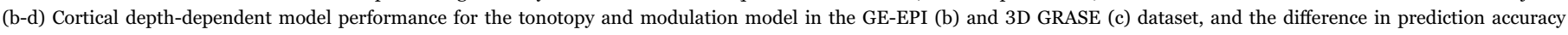

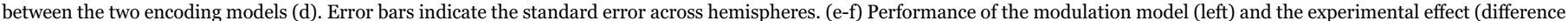

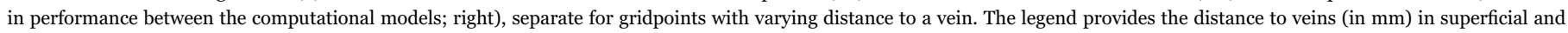
deep cortical depths for each group.

difference in performance between GE-EPI and 3D GRASE was due to the difference in spatial coverage, shown by a reduction in GE-EPI prediction accuracy when coverage was equalized across datasets. The remaining difference in performance between GE-EPI and 3D GRASE may be driven by the higher CNR of GE-EPI compared to 3D GRASE.

The difference in prediction accuracy between the datasets could also be observed in the cortical depth-dependent decoding analysis. The consistently higher prediction accuracy in GE-EPI confirms that the measured GE-EPI responses to sounds, while driven by large veins, were stable. Moreover, it shows that the GE-EPI responses preserve information on the auditory neuronal population functional preferences. Compared to decoding throughout the volume, cortical depth- dependent explorations suffer from lower power due to a decreased number of data points (i.e., voxels). For such analyses, the gain in prediction accuracy due to the higher PSC of GE-EPI may therefore prove critical. That is, while a 3D GRASE dataset may not perform above chance when computing prediction accuracy per cortical depth because of an insufficient number of voxels or CNR, a GE-EPI dataset may have sufficient power to detect cortical depth-dependent differences in information content (Muckli et al., 2015).

Throughout analyses, stronger responses to the sounds coincided with higher prediction accuracy. One exception can be seen when further exploring the PSC and prediction accuracy across different distances to veins (compare Fig. 2c to Fig. 5e). That is, the difference in 


\section{a GE-EPI}
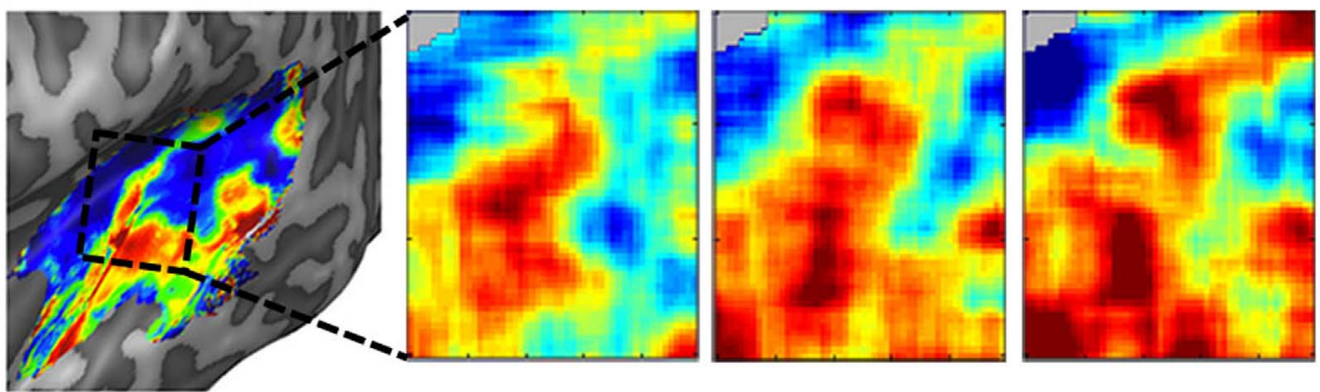

Freq

\section{High}

b 3D GRASE
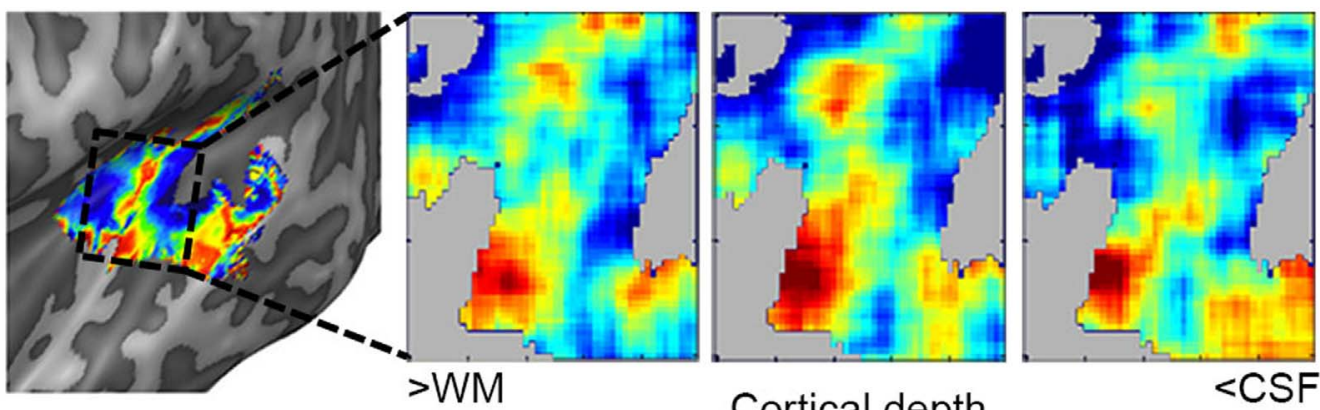

Low

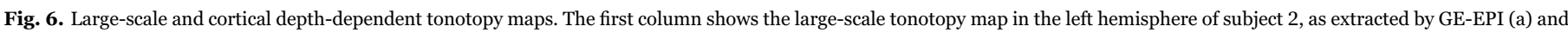

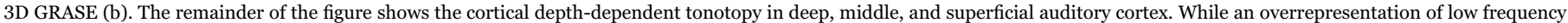
voxels in superficial depths of the GE-EPI dataset can be seen, the preferred frequency content across cortical depths was stable in the 3D GRASE dataset.
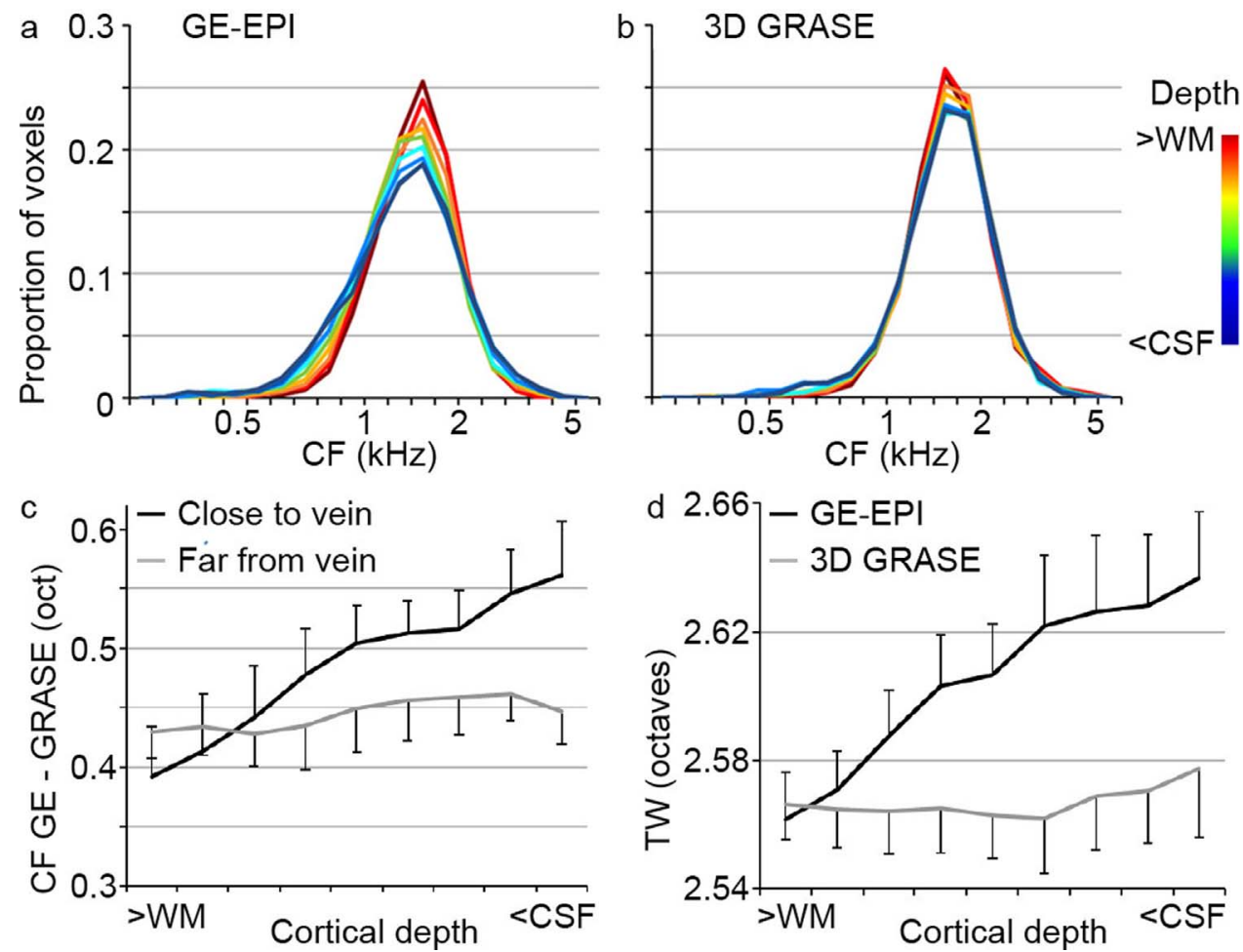

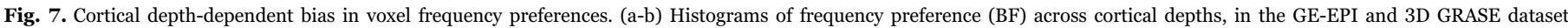

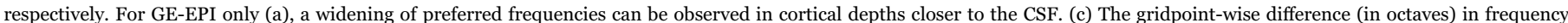

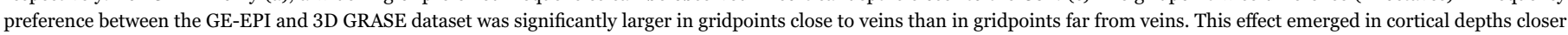

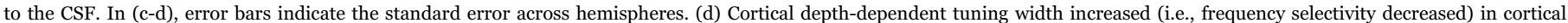
depths closer to the CSF for GE-EPI but not for 3D GRASE. 
PSC between the 25\% of gridpoints closest to large veins compared to the rest of the grid (compare the red line in Fig. $2 \mathrm{c}$ to the other lines) is relatively larger than the corresponding difference in prediction accuracy (Fig. 5e). This may hint at the presence of a trade-off between higher PSC in the GE-EPI dataset, and lower noise and possibly higher spatial specificity in the 3D GRASE dataset.

\section{The effect of unspecific signals on decoding}

While the higher prediction accuracy provided by GE-EPI is valuable, GE-EPI datasets suffer from a large drawback. GE-EPI decoding is based on stable, but spatially unspecific signals (Yacoub et al., 2007). Thus, if the goal is to link the observed decoding effect to a cortical location or depth, these spatially unspecific signals will hamper the interpretation. That is, unspecific signals may change the real spatial variation of decoding. A large vein may carry information outside of its original cortical location (Formisano and Kriegeskorte, 2012; Kriegeskorte et al., 2010; Vu and Gallant, 2015; Yacoub et al., 2007). In the current study, large veins induced higher PSC at the GM surface, and GE-EPI prediction accuracies correspondingly displayed a bias (i.e., increase) towards the GM surface. The increased performance of a model towards the GM-CSF boundary could thus not be interpreted.

Leveraging the higher CNR of GE-EPI could be beneficial if the contribution of large and unspecific veins could be disregarded. There is no straightforward solution to this problem. Previous studies have taken various approaches to ameliorate the influence of unspecific veins on a GE-EPI dataset. A first approach is to exclude those voxels that are closest to large veins. The superficial cortical layers may be excluded altogether, or venous voxels may be identified and excluded (Koopmans et al., 2010; Lu et al., 2010). Our results show that this approach does not solve the problem, as voxels throughout cortical depth (and outside venous voxels) are biased by their proximity to large veins. In fact, removing such voxels may create a problem, as it will produce gaps in the dataset (possibly decreasing the ability to interpret spatial patterns throughout cortical depth). Second, techniques can be used to suppress the BOLD signal of large veins after acquisition. Based on the complex fMRI signal, both magnitude and phase, these methods estimate and remove the part of the signal that originated from macrovasculature (Menon, 2002; Vu and Gallant, 2015). It would be of interest to explore the extent to which these techniques can remove the cortical depth-dependent increase of PSC in submillimeter GE-EPI datasets, and if their employment would improve the specificity of GEEPI datasets (i.e., improve correspondence between cortical depthdependent maps as measured by GE-EPI and 3D GRASE). Third, if information regarding the stimulus and the expected response patterns is available, such a priori information may be used to identify voxels with spatially non-specific responses (Muckli et al., 2015). However, as a priori information on the spatial organization of the cortical stimulus representation is often not available, this technique is not applicable in the general case.

Here, we propose an alternative post-processing technique. That is, our results suggest that while cortical depth-dependent performance of a single condition cannot be interpreted, the difference in cortical depth-dependent prediction accuracy between models is unbiased (see Fig. 5d). Caution must be taken in this approach, as not any differential effect is safe (Polimeni et al., 2010; Vu and Gallant, 2015). The comparison between conditions must be very tight, ensuring that the conditions are affected in the same manner by the macrovasculature. Model comparison is uniquely suited for dealing with unspecific GEEPI signals by differential analysis, as both models use the same GEEPI data as input. This ensures that both conditions (i.e., models) are equally affected by differences in the presence of, and distance to, large veins. Our results confirm this, as the difference between model performances was unbiased, interpretable, and in complete accordance with results from the 3D GRASE dataset.
Spatial specificity is crucial for cortical depth-dependent feature maps

Throughout the auditory pathway, neuronal populations can be characterized by the sound frequency to which they respond best (their best frequency $[\mathrm{BF}]$ ). At each processing stage of the auditory hierarchy, neuronal populations are spatially ordered according to their BF resulting in one or multiple tonotopic maps. At the level of the auditory cortex, tonotopic maps are organized on a relatively large spatial scale. Consequently, tonotopic maps can be delineated with sufficient accuracy also at $3 \mathrm{~T}$ (Moerel et al., 2012; Saenz and Langers, 2014). Beyond this large-scale tonotopic organization, invasive animal studies suggest that additional topographic organizations may be in place at a finer spatial scale. For example, invasive studies throughout species showed that frequency preference is constant perpendicular to the cortical surface resulting in 'frequency columns' (Abeles and Goldstein Jr., 1970; Shamma et al., 1993; Shen et al., 1999; Sugimoto et al., 1997). To explore the stability of frequency preference throughout the cortical depth non-invasively, as was recently achieved using $\mathrm{T}_{2}$ weighted fMRI (3D GRASE; De Martino et al., 2015), an unbiased high specificity feature tuning estimate per cortical depth was necessary.

We evaluated mapping frequency preference across datasets both in a less challenging setting (averaged across cortical depths) and in a situation demanding high specificity (cortical depth-dependent tonotopic maps). The large-scale tonotopic distribution was similar across datasets, and the small differences in tonotopic maps that existed did not hamper the detection of the major tonotopic gradient. Given its larger spatial coverage and sensitivity, GE-EPI dataset is preferable in studies that aim to delineate the large-scale tonotopic map in human auditory cortex. Instead, consistent with results from visual cortex (De Martino et al., 2013b; Yacoub et al., 2007), GE-EPI cortical depthdependent tonotopic maps were biased both in the assigned preferred frequency of a location, and in the selectivity of their frequency preference. Preference biases were observed in middle and superficial (but not deep) cortical depths, in accordance with reports of higher GEEPI specificity close to the WM (De Martino et al., 2013b; Muckli et al., 2015; Nasr et al., 2016; Polimeni et al., 2010).

The vascular contamination in GE-EPI cortical depth-dependent topographic maps reported here and previously (De Martino et al., 2013b; Nasr et al., 2016; Yacoub et al., 2007) may be detrimental to the planned analysis. For example, Yacoub et al. (2007) showed that cortical columns as estimated by $\mathrm{T}_{2}$ and $\mathrm{T}_{2}{ }^{*}$ weighted acquisitions were in accordance with each other only in parts of the visual cortex. Further, recent work by Nasr et al. (2016) acknowledged that the surface contamination may have even artificially "enhanced" the columnar organization in their data. Our data corroborate these findings, showing that the large vein effect in GE-EPI data penetrates to the deepest layers. Therefore, mapping columnar organizations or assigning voxel feature preference using GE-EPI data is compromised irrespective of cortical depth location.

To summarize, when the goal of a study is to observe cortical feature preference organized at a fine spatial scale, deep WM GE-EPI data is preferable over GE-EPI data at more superficial cortical depths. Studies that aim to evaluate the stability and variability of feature preference throughout cortical depth (e.g., to evaluate the presence of a columnar organization, or transformations of stimulus representations throughout the cortical depth) cannot exclude middle and superficial GM from the analysis and, consequently, the acquisition of a $\mathrm{T}_{2}$ weighted datasets or multiple experimental conditions to remove the large vein bias effect is preferable if the continuity and integrity of the maps is to be preserved.

\section{Conclusions}

At ultra-high field, the choice of sequence and acquisition parameters should depend on the planned analyses and/or the nature of the 
neuroscience question being asked. While encoding and decoding profit from the large coverage and high signal in GE-BOLD acquisitions, cortical depth dependent investigations using $\mathrm{T}_{2}{ }^{*}$ weighted images must be based on differential comparisons between conditions that are equally influenced by the macrovasculature. Such differential analysis has the potential to suppress the cortical depth dependent bias present in resulting prediction accuracies. For assigning feature preference on the submillimeter scale, a $\mathrm{T}_{2}$ weighted acquisition is preferable.

\section{Author contributions}

M.M., F.D.M., E.F., and E.Y. designed the experiment. M.M., F.D.M., S.S., A.V., and E.Y. collected the data. M.M. analyzed the data. All authors contributed to writing the manuscript.

\section{Acknowledgements}

This work was supported by the Netherlands Organization for Scientific Research (NWO; Rubicon Grant 446-12-010 to M.M., Veni Grant 451-15-012 to M.M., VIDI Grant 864-13-012 to F.D.M., and VICI grant 453-12-002 to E.F.), the National Institutes of Health (NIH grants P41 EB015894, P30 NS076408, and S10 RR026783), European Research Council (ERC grant number 269853), and the WM KECK Foundation. This research has been made possible with the support of the Dutch Province of Limburg.

\section{Appendix A. Supporting information}

Supplementary data associated with this article can be found in the online version at doi:10.1016/j.neuroimage.2017.03.063.

\section{References}

Abeles, M., Goldstein, M.H., Jr., 1970. Functional architecture in cat primary auditory cortex: columnar organization and organization according to depth. J. Neurophysiol. 33, $172-187$.

Ahveninen, J., Hamalainen, M., Jaaskelainen, I.P., Ahlfors, S.P., Huang, S., Lin, F.-H. Raij, T., Sams, M., Vasios, C.E., Belliveau, J.W., 2011. Attention-driven auditory cortex short-term plasticity helps segregate relevant sounds from noise. Proc. Natl. Acad. Sci. USA 108, 4182-4187. http://dx.doi.org/10.1073/pnas.1016134108.

Cheung, S.W., Bedenbaugh, P.H., Nagarajan, S.S., Schreiner, C.E., 2001. Functional organization of squirrel monkey primary auditory cortex: responses to pure tones. $\mathrm{J}$ Neurophysiol. 85, 1732-1749.

Chi, T., Ru, P., Shamma, S.A., 2005. Multiresolution spectrotemporal analysis of complex sounds. J. Acoust. Soc. Am. 118, 887-906. http://dx.doi.org/10.1121/1.1945807.

Da Costa, S., van der Zwaag, W., Marques, J.P., Frackowiak, R.S.J., Clarke, S., Saenz, M., 2011. Human primary auditory cortex follows the shape of Heschl's gyrus. J. Neurosci. 31, 14067-14075. http://dx.doi.org/10.1523/JNEUROSCI.200011.2011.

De Martino, F., Zimmermann, J., Muckli, L., Ugurbil, K., Yacoub, E., Goebel, R., 2013b. Cortical depth dependent functional responses in humans at $7 \mathrm{~T}$ : improved specificity with 3D GRASE. PLoS One 8, e60514. http://dx.doi.org/10.1371/ journal.pone.0060514.

De Martino, F., Moerel, M., Ugurbil, K., Goebel, R., Yacoub, E., Formisano, E., 2015. Frequency preference and attention effects across cortical depths in the human primary auditory cortex. Proc. Natl. Acad. Sci. USA. http://dx.doi.org/10.1073/ pnas. 1507552112

De Martino, F., Moerel, M., van de Moortele, P.-F., Ugurbil, K., Goebel, R., Yacoub, E., Formisano, E., 2013a. Spatial organization of frequency preference and selectivity in the human inferior colliculus. Nat. Commun. 4, 1386. http://dx.doi.org/10.1038/ ncomms2379.

Douglas, R.J., Martin, K.A.C., 2004. Neuronal circuits of the neocortex. Annu. Rev. Neurosci. 27, 419-451. http://dx.doi.org/10.1146/ annurev.neuro.27.070203.144152.

Duong, T.Q., Yacoub, E., Adriany, G., Hu, X., Kim, S., 2003. Microvascular BOLD Contribution at 4 and $7 \mathrm{~T}$ in the Human Brain: Gradient-echo and Spin-Echo fMRI with Suppression of Blood Effects. 1027, pp. 1019-1027. http://dx.doi.org/10. $1002 / \mathrm{mrm} .10472$.

Duvernoy, H.M., Delon, S., Vannson, J.L., 1981. Cortical blood vessels of the human brain. Brain Res. Bull. 7, 519-579. http://dx.doi.org/10.1016/0361-9230(81) 90007-1.

Feinberg, D.A., Harel, N., Ramanna, S., Ugurbil, K., Yacoub, E., 2008. Sub-millimeter single-shot 3D GRASE with inner volume selection for T2-weighted fMRI applications at 7 T. In: Proceedings of the 16th Annual Meeting of the International Society for Magnetic Resonance in Medicine. Toronto, Canada.
Formisano, E., Kriegeskorte, N., 2012. Seeing patterns through the hemodynamic veil the future of pattern-information fMRI. NeuroImage. http://dx.doi.org/10.1016/ j.neuroimage.2012.02.078.

Formisano, E., Kim, D.-S., Di Salle, F., van De Moortele, P.-F., Ugurbil, K., Goebel, R., 2003. Mirror-symmetric tonotopic maps in human primary auditory cortex. Neuron 40, 859-869. http://dx.doi.org/10.1016/S0896-6273(03)00669-X.

Goebel, R., Esposito, F., Formisano, E., 2006. Analysis of functional image analysis contest (FIAC) data with brainvoyager QX: from single-subject to cortically aligned group general linear model analysis and self-organizing group independent component analysis. Hum. Brain Mapp. 27, 392-401. http://dx.doi.org/10.1002/ hbm.20249.

Goense, J.B.M., Zappe, A.C., Logothetis, N.K., 2007. High-resolution fMRI of macaque V1. Magn. Reson. Imaging 25, 740-747. http://dx.doi.org/10.1016/ j.mri.2007.02.013.

Harel, N., Lin, J., Moeller, S., Ugurbil, K., Yacoub, E., 2006. Combined imaginghistological study of cortical laminar specificity of fMRI signals. Neuroimage 29, 879-887. http://dx.doi.org/10.1016/j.neuroimage.2005.08.016.

Hoerl, A.E., Kennard, R.W., 1970a. Ridge regression: biased estimation for nonorthogonal problems. Technometrics 12, 55-67. http://dx.doi.org/10.1080/ 00401706.1970.10488634.

Hoerl, A.E., Kennard, R.W., 1970b. Ridge regression: applications to nonorthogonal problems. Technometrics 12, 69-82. http://dx.doi.org/10.2307/1267351.

Horton, J.C., Adams, D.L., 2005. The cortical column: a structure without a function Philos. Trans. R. Soc. B Biol. Sci. 360, 837-862. http://dx.doi.org/10.1098/ rstb.2005.1623.

Imaizumi, K., Priebe, N.J., Crum, P. a C., Bedenbaugh, P.H., Cheung, S.W., Schreiner, C.E., 2004. Modular functional organization of cat anterior auditory field. J. Neurophysiol. 92, 444-457. http://dx.doi.org/10.1152/jn.01173.2003.

Jones, S.E., Buchbinder, B.R., Aharon, I., 2000. Three-dimensional mapping of cortical thickness using Laplace's equation. Hum. Brain Mapp. 11, 12-32. http://dx.doi.org/ 10.1002/1097-0193(200009)11:1 < 12::AID-HBM20 > 3.0.CO;2-K.

Kay, K.N., Naselaris, T., Prenger, R.J., Gallant, J.L., 2008b. Identifying natural images from human brain activity. Nature 452, 352-355. http://dx.doi.org/10.1038/ nature06713.

Kay, K.N., David, S.V., Prenger, R.J., Hansen, K. a, Gallant, J.L., 2008a. Modeling lowfrequency fluctuation and hemodynamic response timecourse in event-related fMRI. Hum. Brain Mapp. 29, 142-156. http://dx.doi.org/10.1002/hbm.20379.

Kay, K.N., Rokem, A., Winawer, J., Dougherty, R.F., Wandell, B. a, 2013. GLMdenoise: a fast, automated technique for denoising task-based fMRI data. Front. Neurosci., 1-15. http://dx.doi.org/10.3389/fnins.2013.00247.

Kemper, V.G., De Martino, F., Yacoub, E., Goebel, R., 2015b. Variable flip angle 3DGRASE for high resolution fMRI at 7 T. Magn. Reson. Med.. http://dx.doi.org/ 10.1002/mrm.25979, (00, N./a-N./a).

Kemper, V.G., De Martino, F., Vu, A.T., Poser, B.A., Feinberg, D.A., Goebel, R., Yacoub, E., 2015a. Sub-millimeter T2 weighted fMRI at $7 \mathrm{~T}$ : comparison of 3D-GRASE and 2D SE-EPI. Front. Neurosci. 9, 163. http://dx.doi.org/10.3389/fnins.2015.00163.

Kok, P., Bains, L.J., van Mourik, T., Norris, D.G., de Lange, F.P., 2016. Selective activation of the deep layers of the human primary visual cortex by top-down feedback. Curr. Biol., 1-6. http://dx.doi.org/10.1016/j.cub.2015.12.038.

Koopmans, P.J., Barth, M., Norris, D.G., 2010. Layer-specific BOLD activation in human V1. Hum. Brain Mapp. 31, 1297-1304. http://dx.doi.org/10.1002/hbm.20936.

Koopmans, P.J., Barth, M., Orzada, S., Norris, D.G., 2011. Multi-echo fMRI of the cortical laminae in humans at 7 T. NeuroImage 56, 1276-1285. http://dx.doi.org/ 10.1016/j.neuroimage.2011.02.042.

Kriegeskorte, N., Cusack, R., Bandettini, P., 2010. How does an fMRI voxel sample the neuronal activity pattern: compact-kernel or complex spatiotemporal filter? NeuroImage 49, 1965-1976. http://dx.doi.org/10.1016/j.neuroimage.2009.09.059.

Larkum, M., 2013. A cellular mechanism for cortical associations: an organizing principle for the cerebral cortex. Trends Neurosci. 36, 141-151. http://dx.doi.org/10.1016/ j.tins.2012.11.006.

Lu, H.D., Chen, G., Tanigawa, H., Roe, A.W., 2010. A motion direction map in macaque V2. Neuron 68, 1002-1013. http://dx.doi.org/10.1016/j.neuron.2010.11.020.

Menon, R.S., 2002. Postacquisition suppression of large-vessel BOLD signals in highresolution fMRI. Magn. Reson. Med. 47, 1-9. http://dx.doi.org/10.1002/ mrm.10041.

Moerel, M., De Martino, F., Formisano, E., 2012. Processing of natural sounds in human auditory cortex: tonotopy, spectral tuning, and relation to voice sensitivity. J. Neurosci. 32, 14205-14216. http://dx.doi.org/10.1523/JNEUROSCI.138812.2012 .

Moerel, M., De Martino, F., Santoro, R., Yacoub, E., Formisano, E., 2015. Representation of pitch chroma by multi-peak spectral tuning in human auditory cortex. NeuroImage 106, 161-169.

Moerel, M., De Martino, F., Santoro, R., Ugurbil, K., Goebel, R., Yacoub, E., Formisano, E., 2013. Processing of natural sounds: characterization of multipeak spectral tuning in human auditory cortex. J. Neurosci. 33, 11888-11898. http://dx.doi.org/ 10.1523/JNEUROSCI.5306-12.2013.

Muckli, L., De Martino, F., Vizioli, L., Petro, L.S., Smith, F.W., Ugurbil, K., Goebel, R., Yacoub, E., 2015. Contextual feedback to superficial layers of V1. Curr. Biol. 25, 2690-2695. http://dx.doi.org/10.1016/j.cub.2015.08.057.

Nasr, S., Polimeni, J.R., Tootell, R.B.H., 2016. Interdigitated color- and disparityselective columns within human visual cortical areas V2 and V3. J. Neurosci., 36 http://dx.doi.org/10.1523/JNEUROSCI.3518-15.2016.

Ogawa, S., Tank, D.W., Menon, R., Ellermann, J.M., Kim, S.G., Merkle, H., Ugurbil, K., 1992. Intrinsic signal changes accompanying sensory stimulation: functional brain mapping with magnetic resonance imaging. Proc. Natl. Acad. Sci USA 89, 5951-5955. http://dx.doi.org/10.1073/pnas.89.13.5951. 
Olman, C.A., Harel, N., Feinberg, D.A., He, S., Zhang, P., Ugurbil, K., Yacoub, E., 2012. Layer-specific fMRI reflects different neuronal computations at different depths in human V1. PLoS One 7, e32536. http://dx.doi.org/10.1371/journal.pone.0032536.

Oshio, K., Feinberg, D. a, 1991. GRASE (Gradient- and spin-echo) imaging: a novel fast MRI technique. Magn. Reson. Med. 20, 344-349. http://dx.doi.org/10.1002/ mrm.1910200219.

Polimeni, J.R., Fischl, B., Greve, D.N., Wald, L.L., 2010. Laminar analysis of 7 T BOLD using an imposed spatial activation pattern in human V1. NeuroImage 52, 1334-1346. http://dx.doi.org/10.1016/j.neuroimage.2010.05.005.

Rakic, P., 2008. Confusing cortical columns. Proc. Natl. Acad. Sci. USA 105, $12099-12100$

Ress, D., Glover, G.H., Liu, J., Wandell, B., 2007. Laminar profiles of functional activity in the human brain. NeuroImage 34, 74-84. http://dx.doi.org/10.1016/ j.neuroimage.2006.08.020.

Saenz, M., Langers, D.R.M., 2014. Tonotopic mapping of human auditory cortex. Hear. Res. 307, 42-52. http://dx.doi.org/10.1016/j.heares.2013.07.016.

Santoro, R., Moerel, M., De Martino, F., Goebel, R., Ugurbil, K., Yacoub, E., Formisano, E., 2014. Encoding of natural sounds at multiple spectral and temporal resolutions in the human auditory cortex. PLoS Comput. Biol. 10, e1003412. http://dx.doi.org/ 10.1371/journal.pcbi.1003412.

Shamma, S. a, Fleshman, J.W., Wiser, P.R., Versnel, H., 1993. Organization of response areas in ferret primary auditory cortex. J. Neurophysiol. 69, 367-383.

Shen, J.-X., Xu, Z.-M., Yao, Y.-D., 1999. Evidence for columnar organization in the auditory cortex of the mouse. Hear. Res. 137, 174-177. http://dx.doi.org/10.1016/ S0378-5955(99)00149-5.

Shmuel, A., Yacoub, E., Chaimow, D., Logothetis, N.K., Ugurbil, K., 2007. Spatiotemporal point-spread function of fMRI signal in human gray matter at $7 \mathrm{~T}$. NeuroImage 35, 539-552. http://dx.doi.org/10.1016/j.neuroimage.2006.12.030.

Siero, J.C.W., Petridou, N., Hoogduin, H., Luijten, P.R., Ramsey, N.F., 2011. Cortical depth-dependent temporal dynamics of the BOLD response in the human brain. J. Cereb. Blood Flow Metab. 31, 1999-2008. http://dx.doi.org/10.1038/ jcbfm.2011.57.

Sugimoto, S., Sakurada, M., Horikawa, J., Taniguchi, I., 1997. Primary Auditory Cortex of Mongolian Gerbils. 112, pp. 175-185.

Uğurbil, K., Toth, L., Kim, D.S., 2003. How accurate is magnetic resonance imaging of brain function? Trends Neurosci. 26, 108-114. http://dx.doi.org/10.1016/S01662236(02)00039-5.

Uludağ, K., Müller-Bierl, B., Uğurbil, K., 2009. An integrative model for neuronal activity-induced signal changes for gradient and spin echo functional imaging.
NeuroImage 48, 150-165. http://dx.doi.org/10.1016/j.neuroimage.2009.05.051.

Van de Moortele, P.-F., Auerbach, E.J., Olman, C., Yacoub, E., Uğurbil, K., Moeller, S., 2009. T1 weighted brain images at $7 \mathrm{~T}$ unbiased for proton density, $\mathrm{T} 2 *$ contrast and $\mathrm{RF}$ coil receive $\mathrm{B} 1$ sensitivity with simultaneous vessel visualization. NeuroImage 46, 432-446. http://dx.doi.org/10.1016/j.neuroimage.2009.02.009.

Vaughan, J.T., Garwood, M., Collins, C.M., Liu, W., DelaBarre, L., Adriany, G., Andersen, P., Merkle, H., Goebel, R., Smith, M.B., Ugurbil, K., 2001. $7\{T\}$ vs. $4\{T\}:\{R F\}$ power, homogeneity, and signal-to-noise comparison in head images. Magn. Reson. Med. 46, 24-30. http://dx.doi.org/10.1002/mrm.1156.

Vu, A.T., Gallant, J.L., 2015. Using a novel source-localized phase regressor technique for evaluation of the vascular contribution to semantic category area localization in BOLD fMRI. Front. Neurosci. 9, 1-13. http://dx.doi.org/10.3389/fnins.2015.00411.

Yacoub, E., Harel, N., Ugurbil, K., 2008. High-field fMRI unveils orientation columns in humans. Proc. Natl. Acad. Sci. USA 105, 10607-10612. http://dx.doi.org/10.1073/ pnas.0804110105.

Yacoub, E., Moortele, P.-F. Van De, Shmuel, A., Uğurbil, K., 2005. Signal and noise characteristics of Hahn SE and GE BOLD fMRI at $7 \mathrm{~T}$ in humans. NeuroImage 24, 738-750. http://dx.doi.org/10.1016/j.neuroimage.2004.09.002.

Yacoub, E., Shmuel, A., Logothetis, N., Uğurbil, K., 2007. Robust detection of ocular dominance columns in humans using Hahn Spin Echo BOLD functional MRI at 7 T. NeuroImage 37, 1161-1177. http://dx.doi.org/10.1016/j.neuroimage.2007.05.020.

Yacoub, E., Duong, T.Q., Van De Moortele, P.-F., Lindquist, M., Adriany, G., Kim, S.-G., Uğurbil, K., Hu, X., 2003. Spin-echo fMRI in humans using high spatial resolutions and high magnetic fields. Magn. Reson. Med. 49, 655-664. http://dx.doi.org/ 10.1002/mrm.10433.

Yacoub, E., Shmuel, a, Pfeuffer, J., Moortele, Van De, Adriany, P.F., Andersen, G., Vaughan, P., Merkle, J.T., Ugurbil, H., Hu, X, K., 2001. Imaging brain function in humans at 7 T. Magn. Reson. Med. 45, 588-594. http://dx.doi.org/10.1002/ mrm.1080.

Yu, X., He, Y., Wang, M., Merkle, H., Dodd, S.J., Silva, A.C., Koretsky, A.P., 2016. Sensory and optogenetically driven single-vessel fMRI. Nat. Methods 13, 337-340. http://dx.doi.org/10.1038/nmeth.3765.

Zhao, F., Wang, P., Kim, S., 2004. Cortical depth-dependent gradient-echo and spin-echo BOLD fMRI at 9.4 T. Magn. Reson. Med. 51, 518-524. http://dx.doi.org/10.1002/ mrm.10720.

Zimmermann, J., Goebel, R., De Martino, F., van de Moortele, P.-F., Feinberg, D., Adriany, G., Chaimow, D., Shmuel, A., Uğurbil, K., Yacoub, E., 2011. Mapping the organization of axis of motion selective features in human area MT using high-field fMRI. PLoS One 6, e28716. http://dx.doi.org/10.1371/journal.pone.0028716. 